\title{
Zirconosilicates in the kakortokites of the llímaussaq complex, South Greenland: Implications for fluid evolution and high-field-strength and rare-earth element mineralization in agpaitic systems
}

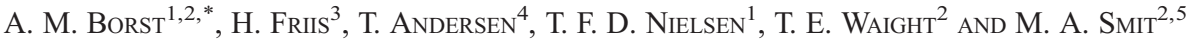 \\ 1 Department of Petrology and Economic Geology, Geological Survey of Denmark and Greenland, Øster Voldgade 10, \\ 1350 Copenhagen, Denmark \\ 2 Department of Geosciences and Natural Resource Management (Geology Section), University of Copenhagen, \\ Øster Voldgade 10, 1350, Copenhagen, Denmark \\ 3 Natural History Museum, University of Oslo, P.O. 1172 Blindern, N-0318, Oslo, Norway \\ 4 Department of Geosciences, University of Oslo, P.O. 1047 Blindern, N-0316, Oslo, Norway \\ 5 Department of Earth, Ocean and Atmospheric Sciences, University of British Columbia, 6339 Stores Road, \\ Vancouver, V6 T 1Z4, Canada
}

[Received 7 April 2015; Accepted 25 June 2015; Associate Editor: Kathryn Goodenough]

\section{ABSTRACT}

The layered agpaitic nepheline syenites (kakortokites) of the Ilímaussaq complex, South Greenland, host voluminous accumulations of eudialyte-group minerals (EGM). These complex Na-Ca-zirconosilicates contain economically attractive levels of $\mathrm{Zr}, \mathrm{Nb}$ and rare-earth elements ( $R E E)$, but have commonly undergone extensive autometasomatic/hydrothermal alteration to a variety of secondary mineral assemblages. Three EGM alteration assemblages are recognized, characterized by the secondary zirconosilicates catapleiite, zircon and gittinsite. Theoretical petrogenetic grid models are constructed to assess mineral stabilities in terms of component activities in the late-stage melts and fluids. Widespread alteration of EGM to catapleiite records an overall increase in water activity, and reflects interaction of EGM with late-magmatic Na-, Cl- and F-rich aqueous fluids at the final stages of kakortokite crystallization. Localized alteration of EGM and catapleiite to the rare $\mathrm{Ca}-\mathrm{Zr}$ silicate gittinsite, previously unidentified at Ilímaussaq, requires an increase in $\mathrm{CaO}$ activity and suggests post-magmatic interaction with $\mathrm{Ca}-\mathrm{Sr}$ bearing aqueous fluids. The pseudomorphic replacement of EGM in the kakortokites was not found to be associated with significant remobilization of the primary $\mathrm{Zr}, \mathrm{Nb}$ and $R E E$ mineralization, regardless of the high concentrations of potential transporting ligands such as $\mathrm{F}$ and $\mathrm{Cl}$. We infer that the immobile behaviour essentially reflects the neutral to basic character of the late-magmatic fluids, in which $R E E$-F compounds are insoluble and remobilization of $R E E$ as $\mathrm{Cl}$ complexes is inhibited by precipitation of nacareniobsite-(Ce) and various $\mathrm{Ca}-R E E$ silicates. A subsequent decrease in $\mathrm{F}^{-}$activity would furthermore restrict the mobility of $\mathrm{Zr}$ as hydroxyl-fluoride complexes, and promote precipitation of the secondary zirconosilicates within the confines of the replaced EGM domains.

KEYworDS: Ilímaussaq, late-magmatic alteration, eudialyte, catapleiite, gittinsite, HFSE and REE mineralization, nepheline syenites, chemographic analysis, agpaitic systems.

\section{Introduction}

* E-mail: anoukborst@gmail.com

DOI: $10.1 \overline{180 / \mathrm{minmag} .2016 .080 .046}$
BASED on studies of the peralkaline Ilímaussaq complex, South Greenland, Ussing (1912) introduced the term agpaite to describe nepheline 
syenites with molar $(\mathrm{Na}+\mathrm{K}) / \mathrm{Al}$ ratios greater than 1.2. Agpaitic rocks are additionally characterized by the presence of complex $\mathrm{Na}-\mathrm{Zr}$-Ti silicates, such as eudialyte-group minerals (EGM), aenigmatite and rinkite, instead of the common $\mathrm{Zr}$-Ti phases, zircon and ilmenite (e.g. Sørensen, 1997; LeMaitre, 2003; Marks et al., 2011). The magmas that produce these unusual assemblages typically form in rift-related settings and are characterized by extreme enrichments in alkalis, halogens $(\mathrm{F}, \mathrm{Cl}, \mathrm{Br})$ and high-field-strength elements (HFSE), e.g. Zr, $\mathrm{Ti}, \mathrm{Nb}, \mathrm{Ta}$ and $R E E$ at economically attractive levels (e.g. Sørensen, 1992; Bailey et al., 2001).

Agpaitic melts generally crystallize over extended temperature intervals (c. 1000 to $450^{\circ} \mathrm{C}$ ) under reduced conditions (e.g. Markl et al., 2001; 2010). Low oxygen fugacity is inferred to suppress segregation of aqueous fluids enriched in $\mathrm{Na}, \mathrm{F}$ and $\mathrm{Cl}$ and other water-soluble elements from the evolving melt until near-solidus temperatures (e.g. Konnerup-Madsen et al., 2001; Markl et al., 2001). This enables the melt to evolve to highly sodic and volatile-rich compositions, enhancing the solubility of HFSE in the melt until their sequestration in the characteristic Na- and HFSE-rich agpaitic minerals (e.g. Sørensen et al., 1997; Andersen et al., 2010; Marks et al., 2011). In the final stages of crystallization, the primary minerals are commonly subjected to intense auto-metasomatic alteration and hydrothermal overprinting (e.g. Markl et al., 2001; Mitchell and Liferovich, 2006; Marks and Markl, 2015). In many peralkaline complexes, such processes led to significant remobilization of the primary HFSE and REE mineralization (e.g. Boily and Williams-Jones, 1994; Salvi and WilliamsJones, 1995; 1996; 2005; 2006; Salvi et al., 2000; Sheard et al., 2012; Gysi and Williams-Jones, 2013; Estrade et al., 2015).

With a growing demand and potential supplyrisk for HFSE and REE in the high-tech industry (Roskill, 2011), peralkaline and agpaitic complexes are considered as important alternatives to more commonly exploited resources for these elements, such as carbonatites and heavy-mineral sands (Chakhmouradian and Zaitsev, 2012). This has also sparked an increased demand for better characterization of the magmatic and subsequent hydrothermal petrogenesis of these deposits and their often complex mineralogy. To contribute to the progress in this field, we characterize EGM alteration assemblages from the agpaitic nepheline syenites of the Mesoproterozoic Ilímaussaq complex; the type locality for e.g. agpaites (Ussing, 1912), eudialyte s.l. (Stromeyer, 1819) and rinkite (Lorenzen, 1884).
Alternating layers of arfvedsonite-, EGM-, and feldspar-rich nepheline syenites, known as the kakortokites, in the lower part of the well-preserved and exposed Ilímaussaq magma chamber host large volumes of cumulus EGM (general formula $\mathrm{Na}_{15}(\mathrm{Ca}, R E E)_{6}(\mathrm{Fe}, \mathrm{Mn})_{3} \mathrm{Zr}_{3} \mathrm{Si}\left(\mathrm{Si}_{25} \mathrm{O}_{72}\right)\left(\mathrm{O}, \mathrm{OH}, \mathrm{H}_{2}\right.$ $\mathrm{O})_{3}(\mathrm{Cl}, \mathrm{OH})_{2}$, Johnsen et al., 2003). The latter account for most of the exploitable $\mathrm{Zr}, \mathrm{Nb}$ and $R E E$ mineralization within the kakortokites, and can readily be extracted by magnetic separation (e.g. Bohse et al., 1971; Sørensen, 1992; Zakharov et al., 2011). Wide-spread alteration of EGM, however, led to the formation of complex pseudomorphic aggregates of secondary zirconosilicates, aluminosilicates and various $\mathrm{Nb}$ and $R E E$ phases. The alteration effectively fractionates the HFSE and $R E E$ among the secondary mineral phases and has unconstrained consequences for the bulk-rock concentrations and economic potential of the kakortokites.

Previous studies have shown two distinct alteration types after EGM in the kakortokites and associated pegmatites, either dominated by zircon or catapleiite (e.g. Ussing, 1912; Graser and Markl, 2008; Karup-Møller et al., 2010; Karup-Møller and Rose-Hansen, 2013;). We report a third and new EGM alteration assemblage, containing the rare $\mathrm{Ca}$ zirconosilicate gittinsite $\left(\mathrm{CaZrSi}_{2} \mathrm{O}_{7}\right)$. Gittinsite was first described in association with vlasovite and EGM from the Kipawa complex in Canada (Ansell, 1980); to our knowledge, the only known occurrence of gittinsite in agpaitic nepheline syenites. More often gittinsite is found in lowtemperature alteration assemblages with $\mathrm{Zr}$ phases such as elpidite, armstrongite and zircon in Sisaturated peralkaline granites, such as Khan Bogd and Khaldzan Buregte in Mongolia (e.g. Kynicky et al., 2011; Kempe et al., 1999), Strange Lake and Thor Lake in Canada (e.g. Birkett et al., 1992; Salvi and Williams-Jones, 1995, 1996; Sheard et al., 2012) and additionally in carbonatites of the Afrikanda complex in Russia (Chakhmouradian and Zaitsev, 2002). Crystallization of the Ca-mineral gittinsite seems unusual in the highly persodic syenites of Ilímaussaq, which generally display Ca-depletion with magmatic evolution (Marks et al., 2011). Based on the observed EGM alteration assemblages, we constructed theoretical chemographic grid models to assess the physicochemical conditions which control EGM replacement reactions. Mass- and volume-relations are discussed to identify bulk loss and gain of components. Together, these efforts contribute to the understanding of late-magmatic and 
hydrothermal fluid evolution in the kakortokites and their potential impact on HFSE and REE mineralization in agpaitic complexes.

\section{Geological Setting}

The Ilímaussaq complex (1160 \pm 5 Ma; Waight et al., 2002; Krumrei et al., 2006) is part of the Mesoproterozoic Gardar Province, South Greenland (Upton, 2013). The complex was emplaced during at least four intrusive pulses successively crystallizing augite syenites, alkali granites, quartz syenites and the volumetrically dominant agpaitic nepheline syenites (see reviews in Larsen and Sørensen, 1987; Sørensen, 2001; Sørensen et al., 2006; Marks and Markl, 2015). The agpaitic sequence (c. $1500 \mathrm{~m}$ thick) consists of: (1) an upper series of downward-crystallized roof cumulates (pulaskite, foyaite, sodalite foyaite and naujaite); (2) a lower series of rhythmically layered floor cumulates (kakortokites); and (3) a 'sandwich horizon' of lujavrites that represent the most evolved melt fractions (e.g. Ussing, 1912; Ferguson, 1964; Sørensen et al., 2006). All agpaitic units are textural varieties of nepheline syenites with varying proportions of the liquidus phases alkali feldspar, nepheline, sodalite, aegirine, arfvedsonite and EGM. Common intercumulus and accessory minerals are aenigmatite, rinkite-group minerals, fluorite, apatite and analcime.

The present work focuses on the kakortokitelujavrite sequence exposed at Kringlerne in the southern part of the complex (Fig. 1). The lower $\sim 200 \mathrm{~m}$ of the kakortokite sequence exhibit strong magmatic layering (lower layered kakortokites or LLK), comprising at least 29 triple-layered units (Fig. 2). Each unit consists of a basal arfvedsoniterich layer (black), a thin EGM-rich layer (red, sometimes absent) and an upper white layer rich in nepheline and feldspar (e.g. Bohse et al., 1971; Larsen and Sørensen, 1987). The units are numbered from -11 to +16 , relative to the welldeveloped marker horizon of unit 0 . The layering steepens towards the margins of the intrusion and gradually disappears into a marginal pegmatite zone of the kakortokite (up to $100 \mathrm{~m}$ wide; Sørensen et al., 2006), ending with a sharp intrusive contact against the earlier augite syenite (Fig. 1).

The LLK are conformably overlain by c. $50 \mathrm{~m}$ of weakly-layered and poorly exposed kakortokites (slightly layered kakortokites or SLK). On the northern side of the Lakseelv fault-zone more kakortokites $(\mathrm{c} .60 \mathrm{~m}$ ) are exposed also showing the modal layering similar to the LLK (Fig. 1). These 'transitional layered kakortokites' (TLK) are labelled downwards from A to F and are characterized by an upwards decrease in grain size and modal amount of arfvedsonite relative to aegirine (Bohse and Andersen, 1981). The TLK gradually evolve into aegirine-dominated lujavrites, which are subdivided into a lower aegirine lujavrite I and an upper, finer-grained aegirine lujavrite II. These are overlain by arfvedsonite-dominated lujavrites. Both the TLK and lujavrites exhibit pronounced magmatic fabrics, indicative of low-viscosity melts and near-solidus to solid state deformation during emplacement (e.g. Sørensen et al., 2006; Ratschbacher et al., 2015). Detailed discussions on the evolution of the kakortokite-lujavrite series are provided by Pfaff et al. (2008), Marks and Markl (2015) and Ratschbacher et al. (2015).

\section{Analytical methods}

The EGM and their pseudomorph assemblages were analysed in samples from units -11 to +16 in the LLK, unit E and B from the TLK and EGM-rich layer II from aegirine lujavrite I. Zirconosilicate compositions were determined by wavelengthdispersive spectroscopy (WDS) using a Cameca SX100 electron probe micro-analyser (EPMA) at the University of Oslo. EGM were analysed with an acceleration voltage of $15 \mathrm{kV}$, a beam current of $15 \mathrm{nA}$ and a spot size of $20-25 \mu \mathrm{m}$ to minimize sample damage and $\mathrm{Na}$ migration. Other zirconosilicates were analysed using a $15 \mathrm{kV}$ acceleration voltage, $10 \mathrm{nA}$ beam current and $10 \mu \mathrm{m}$ beam size, because of their smaller grain size. The elements $\mathrm{Na}$, $\mathrm{Zr}, \mathrm{Si}$ and $\mathrm{Cl}$ were measured first and $\mathrm{X}$-ray counts were monitored in-run for signal stability to prevent migration and associated beam damage effects. Counting times were 10-60 s on peak, and half of that on each baseline, with shorter times for volatile elements. Calibration standards were wollastonite $(\mathrm{Ca} K \alpha, \mathrm{Si} K \alpha)$, albite $(\mathrm{Na} L \alpha)$, corundum $(\mathrm{Al} K \alpha), \mathrm{Fe}$ metal $(\mathrm{Fe} K \alpha)$, MnTi-oxide $(\mathrm{Mn} K \alpha$, Ti $K \alpha)$, orthoclase $(\mathrm{K} K \alpha)$, synthetic alforsite $(\mathrm{Cl} K \alpha)$, Monastery Mine zircon $(\mathrm{Zr} L \alpha)$, Hf metal ( $\mathrm{HfM} \alpha)$, $\mathrm{Nb}$ metal $(\mathrm{Nb} L \alpha)$ and synthetic orthophosphates (Jarosewich and Boatner, 1991) for REE and Y ( $\mathrm{La} L \alpha, \mathrm{Ce} L \alpha$, $\mathrm{Nd} L \beta, Y L \alpha)$. Full-range spectra and energy-dispersive spectral (EDS) analyses were acquired on a few grains prior to analyses, to check for the presence of elements not included in the analyses. Peak and background positions were chosen carefully to avoid interferences from other elements in the 

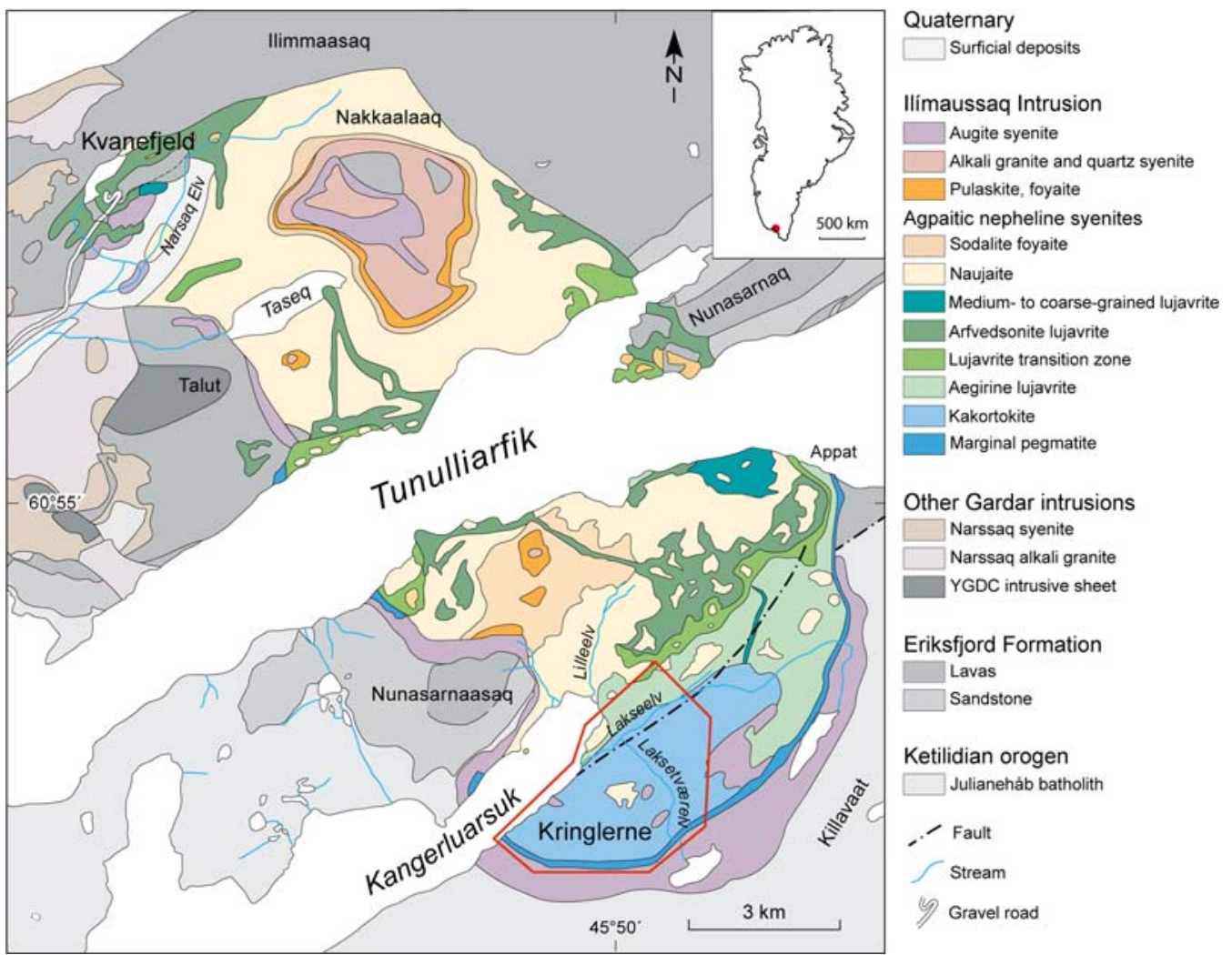

\section{Other Gardar intrusions

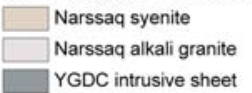

\section{Eriksfjord Formation

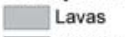 \\ Sandstone}

\section{Ketilidian orogen}

$\square$ Julianeháb batholith

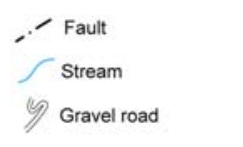

FIG. 1. Geological map of the Ilímaussaq intrusion and surrounding geology from Upton (2013), based on Ferguson (1964), Andersen et al., (1988) and Sørensen (2001). Samples were obtained from the Kringlerne, Lakseelv and Laksetværelv areas in the southern part of the complex (red box).

spectral domains of interest. Discrete pulse-height analysis was applied to further minimize interferences from higher order spectral lines. Matrix corrections were applied according to the PAP procedure (Pouchou and Pichoir, 1984). EGM formulae were calculated based on $(\mathrm{Si}+\mathrm{Zr}+\mathrm{Ti}+$ $\mathrm{Nb}+\mathrm{Al}+\mathrm{Hf})=29$ (Johnsen and Grice, 1999).

Backscattered electron images as well as analyses of aegirine were obtained using a JEOL JXA8200 EPMA at the University of Copenhagen, using an acceleration voltage of $15 \mathrm{kV}$, beam current of $15 \mathrm{nA}$ and beam size of $5 \mu \mathrm{m}$. Counting times and X-ray lines were as described above. Calibration standards deviating from those above are corundum $(\mathrm{Al} K \alpha)$, hematite $(\mathrm{Fe} K \alpha)$, baddelyite $(\mathrm{Zr} L \alpha)$ and rutile $(\mathrm{Ti} K \alpha)$. Matrix corrections were carried out following the $\phi \rho z$ correction protocol. Secondary REE minerals were considered too small $(<5 \mu \mathrm{m})$ for accurate quantitative analyses using WDS, and were analysed semi-qualitatively using EDS.
A Siemens D5005 X-ray diffractometer equipped with a $\mathrm{CuK \alpha}$ tube at the Natural History Museum of Oslo was used for mineral identification. A selection of $\mathrm{Na}$ and $\mathrm{Ca}$ zirconosilicates from the museum collection, i.e. catapleiite, gaidonnayite, gittinsite, lemoynite, parakeldyshite, keldyshite, elpite, armstrongite and vlasovite, were checked by X-ray diffraction (XRD) (using only a small fraction of the grains) and embedded in epoxy to serve as external standards for EPMA analyses. Powders of catapleiite- and gittinsite-bearing pseudomorphs after EGM and a crosscutting gittinsite-bearing vein were extracted for XRD analyses using a diamond-microdrill. The XRD confirmed the presence of catapleiite and excluded the presence of related phases gaiddonayite $\left(\mathrm{Na}_{2} \mathrm{Zr}_{3} \mathrm{SiO}_{9} \cdot 2 \mathrm{H}_{2} \mathrm{O}\right)$ and hilairite $\left(\mathrm{Na}_{2} \mathrm{ZrSi}_{3} \mathrm{O}_{9} \cdot 3 \mathrm{H}_{2} \mathrm{O}\right)$. Moreover, the distinct platy or hexagonal crystal habit characteristic for catapleiite (as opposed to mostly equidimensional habits for gaidonnayite) suggests that catapleiite is the dominant phase in 


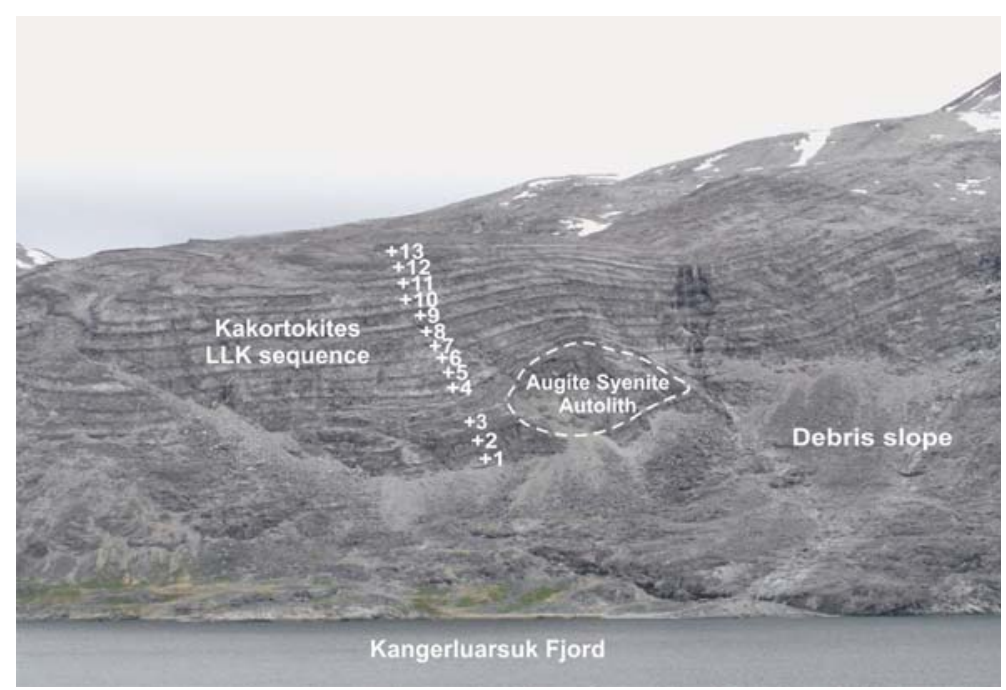

FIG. 2. Photograph of the lower layered kakortokite series (LLK). The cliff is $\sim 300 \mathrm{~m}$ high and is exposed along the south-east coast of the Kangerluarsuk Fjord at Kringlerne. The visible units are numbered +1 to +13 (Bohse and Andersen, 1981). Unit +3 contains a large augite syenite autolith (dashed lines).

most EGM alteration assemblages. It should however be noted that the occurrence of gaiddonayite or hilairite cannot be excluded for samples not checked by XRD, given the difficulty of distinguishing these from catapleiite based on EPMA analyses alone.

\section{Eudialyte-group minerals and their alteration paragenesis}

In the kakortokites and lujavrites EGM occur as euhedral crystals which are part of the early crystallizing assemblage together with alkali feldspar, nepheline and arfvedsonite (e.g. Bohse et al., 1971; Pfaff et al., 2008; Lindhuber et al., 2015, Ratschbacher et al., 2015). Modal proportions of EGM vary from c. 5 to 20 vol.\% in black and white kakortokites and lujavrites and 20 to 40 vol.\% in red kakortokites. Average EGM grain sizes are 1 to $2 \mathrm{~mm}$ in the kakortokites (Fig. $3 a, b$ ) and $\sim 0.3 \mathrm{~mm}$ in the TLK and aegirine lujavrite. Unaltered crystals typically exhibit sector-zoning, submicrometre oscillatory zoning and complex corerim zoning (Fig. $3 a, b$ ). In the majority of samples EGM are partially or completely replaced by secondary assemblages. The degree of alteration ranges from initial replacement along crystal margins and along fractures to complete pseudomorphic replacement (Fig. 3c-h). Pseudomorphs after EGM commonly cluster in zones of intense alteration, but also exist in direct contact with pristine EGM crystals (Fig. 3c,d). The degree of alteration is highly variable throughout the sequence and generally independent of lithology, although, EGM alteration is slightly more pervasive in the TLK and the aegirine lujavrites than in the LLK. Seventy-five percent of the investigated samples $(n=\sim 80)$ exhibit variable degrees of EGM alteration and in $\sim 45 \%$ of the samples the majority of EGM are completely replaced. The degree and type of EGM alteration is generally independent of the surrounding mineral phases, although EGM are locally shielded from alteration when they are poikilitically enclosed by primary arfvedsonite.

Throughout the sequence, most EGM alteration assemblages contain catapleiite-group minerals as the major $\mathrm{Zr}$ phase; aegirine as the major Fe phase; fluorite and pectolite as major Ca phases; analcime, albite or $\mathrm{K}$-feldspar as $\mathrm{Na}$ and $\mathrm{K}$ phases and nacareniobsite-(Ce) as the main $\mathrm{Nb}-R E E$ phase. The $R E E$ are further distributed among micrometresized crystals of monazite, britholite-(Ce) and a related but poorly-defined group of Ca-REE silicates. The latter were studied by Karup-Møller and Rose-Hansen (2013), who described two dominant varieties and referred to them as Ca-rich $\mathrm{A} 1$ and $\mathrm{Ca}$-poor $\mathrm{A} 1$, with the empirical formulae $\mathrm{HCa}_{3} R E E_{6}\left(\mathrm{SiO}_{4}\right)_{6}(\mathrm{~F} \square)$ (where $\square=$ vacancy) and $(\mathrm{Fe}, \mathrm{Mn}, \mathrm{Ca})_{1.5} R E E_{6} \quad \mathrm{Si}_{6} \mathrm{FO}_{22}$, respectively. Both 

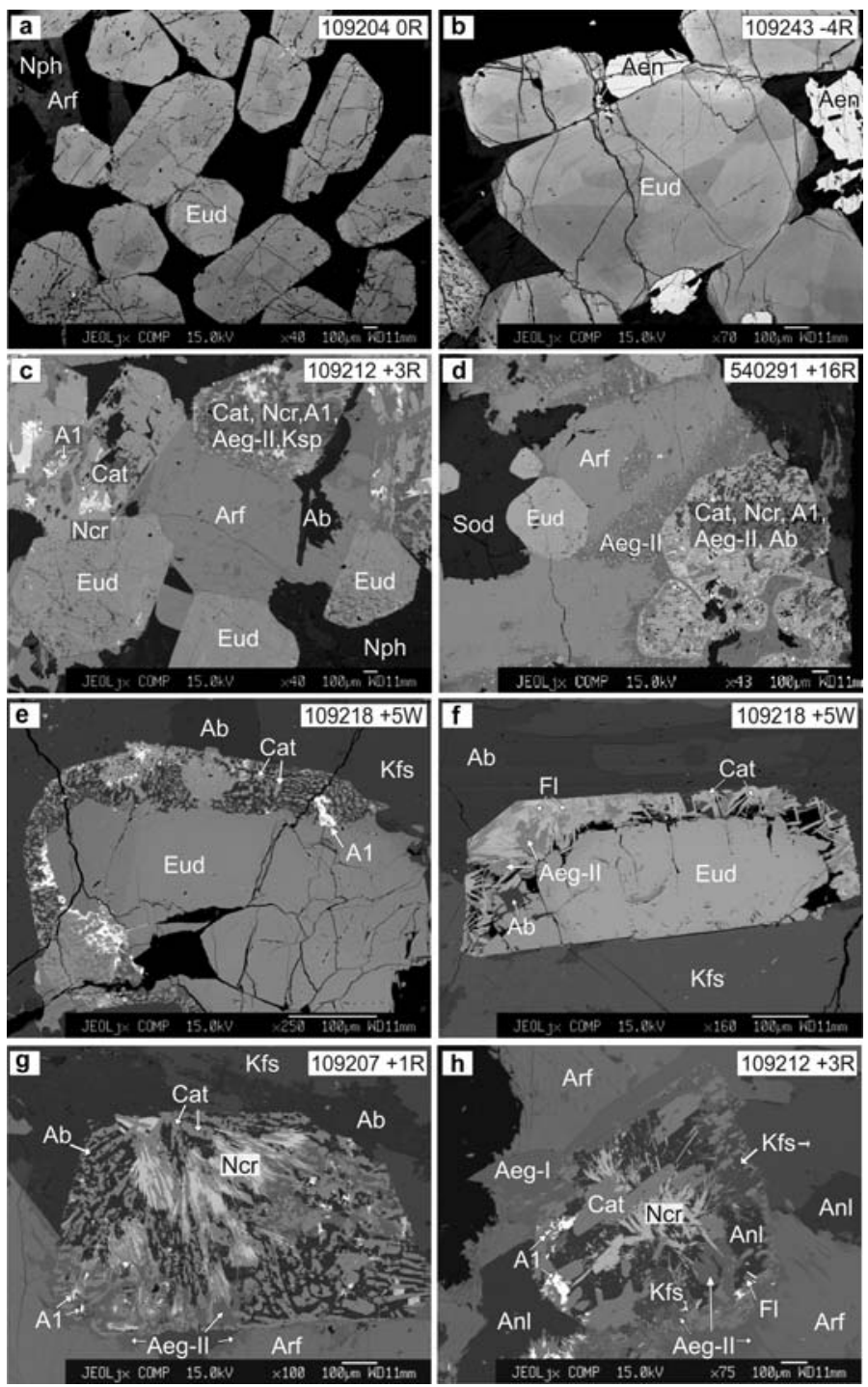

FIG. 3. Selection of backscatter electron images of EGM and their alteration products. Sample and layer number in top right corner. (a) Euhedral EGM crystals showing sector-zoning. (b) Sector- and oscillatory-zoned EGM with aenigmatite, arfvedsonite and nepheline. (c) Decomposed EGM adjacent to an unaltered EGM grain, surrounded by arfvedsonite and interstitial nepheline. (d) Catapleiite dominated (type-I) pseudomorphs after EGM with replacement of adjacent arfvedsonite by secondary aegirine and Mn-Ti phases. (e) Symplectite rim on EGM, consisting of finelyintergrown catapleiite, albite, K-feldspar and the Ca-poor variety of A1. $(f)$ Euhedral EGM with rim altered to catapleiite, aegirine, albite and fluorite. Note the preservation of the original crystal faces. (g) Pseudomorph after EGM containing catapleiite, nacareniobsite-(Ce), aegirine, pectolite, albite, A1 (Ca-rich variety) and minor Nb-oxides. $(h)$ Type-I pseudomorph after EGM containing catapleiite, nacareniobsite-(Ce), aegirine, analcime, K-feldspar and A1 (Capoor variety). Note that both K-feldspar and analcime have grown into the pseudomorph along their original contacts with the precursor eudialyte. Abbreviations: Arf = arfvedsonite, $\mathrm{Nph}=$ nepheline, $\mathrm{Sod}=$ sodalite, Eud = eudialyte, Aen $=$ aenigmatite, $\mathrm{Ab}=$ albite, $\mathrm{Kfs}=\mathrm{K}$-feldspar, $\mathrm{Cat}=$ catapleiite, $\mathrm{Fl}=$ fluorite, Aeg- $\mathrm{I}=$ magmatic aegirine, Aeg-II $=$ secondary aegirine, $\mathrm{Ncr}=$ nacareniobsite- $(\mathrm{Ce}), \mathrm{A} 1=\mathrm{Ca}$-rich or $\mathrm{Ca}$-poor $R E E$ silicate. 

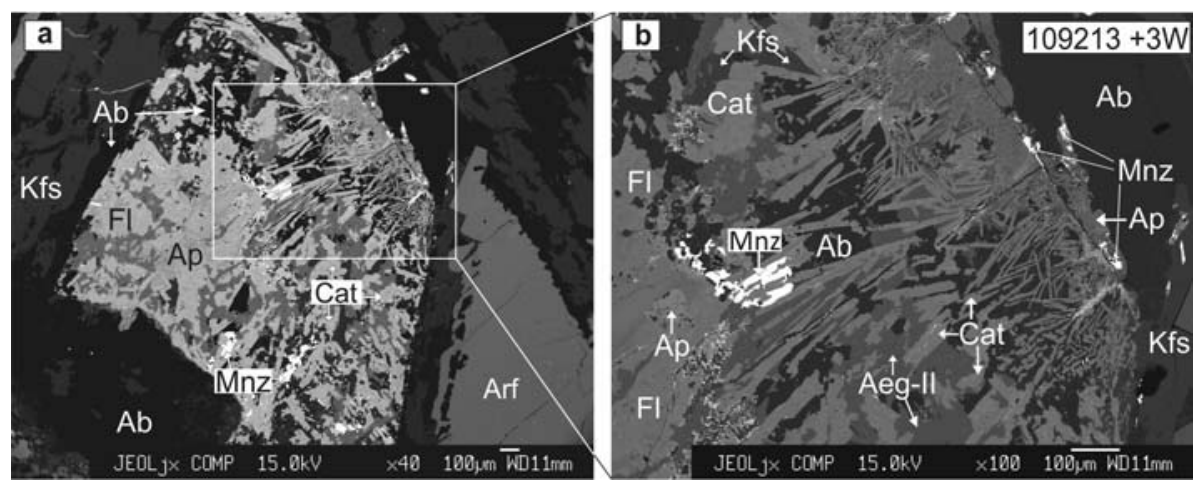

FIG 4. (a) Type-I pseudomorph after EGM in a white kakortokite, containing monazite (Mnz, bright mineral) as the major REE phase, together with abundant fluorapatite (Ap), fluorite and interstitial albite, K-feldspar and aegirine. (b)

Thin laths of catapleiite nucleating from the outer margins of the precursor eudialyte crystal.

varieties were observed in the current sample-set. Some pseudomorph assemblages contain dispersed grains $(<5 \mu \mathrm{m})$ of additional $\mathrm{Nb}$ phases such as pyrochlore, fersmite and fergusonite-(Y).

Previous studies (Ussing, 1912; Karup-Møller et al., 2010) also reported EGM alteration assemblages containing zircon, in addition to catapleiite. These occurrences are limited to extensively altered kakortokites, lujavrites and related pegmatites, i.e. spatially restricted lithologies that were not investigated in the current study. Instead, we describe a third EGM alteration assemblage from the kakortokites, dominated by the rare $\mathrm{Ca}$ zirconosilicate gittinsite. Only the two observed parageneses characterized by catapleiite and gittinsite (type-I and II, respectively) are described in further detail below.

\section{Catapleiite-bearing paragenesis}

Catapleiite-group minerals $\left((\mathrm{Na}, \mathrm{Ca}, \square)_{2} \mathrm{ZrSi}_{3} \mathrm{O}_{9} \cdot 2\right.$ $\mathrm{H}_{2} \mathrm{O}$ ) are the most common alteration products after EGM throughout the complex. At small degrees of alteration, catapleiite replaced EGM along rims and cracks, forming anhedral to subhedral grains, sometimes intimately intergrown with secondary feldspar and/or aegirine (Fig. 3c,d). At larger degrees of replacement, catapleiite occurs as subhedral to euhedral elongate crystals, up to $0.5 \mathrm{~mm}$ long. In some alteration aggregates catapleiite crystals nucleate inwards from the rim of the precursor EGM grain (Fig. 3f, Fig. 4b) and in some coarser grained pseudomorphs the well-developed catapleiite laths are stacked and fully aligned. Aegirine, pectolite, fluorite, various $\mathrm{Nb}$ and $R E E$ phases and occasionally apatite generally form subhedral aggregates between the catapleiite crystals (Fig. 3). The remaining interstitial space is occupied by aluminosilicates, i.e. albite, K-feldspar or analcime, which can occur together in a single pseudomorph (Fig. $3 c-h$, Fig. 4).

\section{Gittinsite-bearing paragenesis}

Gittinsite is the dominant zirconosilicate in EGM pseudomorphs in a sample from layer +3 black (109211). It occurs as subhedral crystals of $<15 \mu \mathrm{m}$ in size, which are intergrown with Ca-rich catapleiite and annite (Fe-biotite, Fig. 5). Textural relations suggest that gittinsite partially or completely replaced catapleiite from a previous EGM alteration paragenesis (Fig. 5b,c,d). Other common phases in the gittinsite-bearing pseudomorphs are analcime, aegirine, fluorite, pectolite, allanite-(Ce), fergusonite-( $\mathrm{Y})$ and $\mathrm{Ca}-R E E$ silicates $\mathrm{A} 1$ and $\mathrm{A} 2$. A few grains of relict EGM are partially replaced by gittinsite, analcime and annite, with no evidence for intermediate catapleiite formation (Fig. $5 f, g, h$ ). Primary aluminosilicates (e.g. feldspar, nepheline and sodalite) are replaced by analcime throughout the sample. Furthermore, a 1-2 mm-wide hydrothermal vein of pectolite, annite and analcime crosscuts the primary mineral assemblage. Aggregates of gittinsite, intergrown with catapleiite, annite and an unidentified Ca-Y-HREE phase were found inside the hydrothermal vein, in close proximity of a fragmented precursor EGM crystal. In a separate sample (layer 0 red), an isolated crystal of gittinsite was identified within a catapleiite-dominated pseudomorph after EGM, 

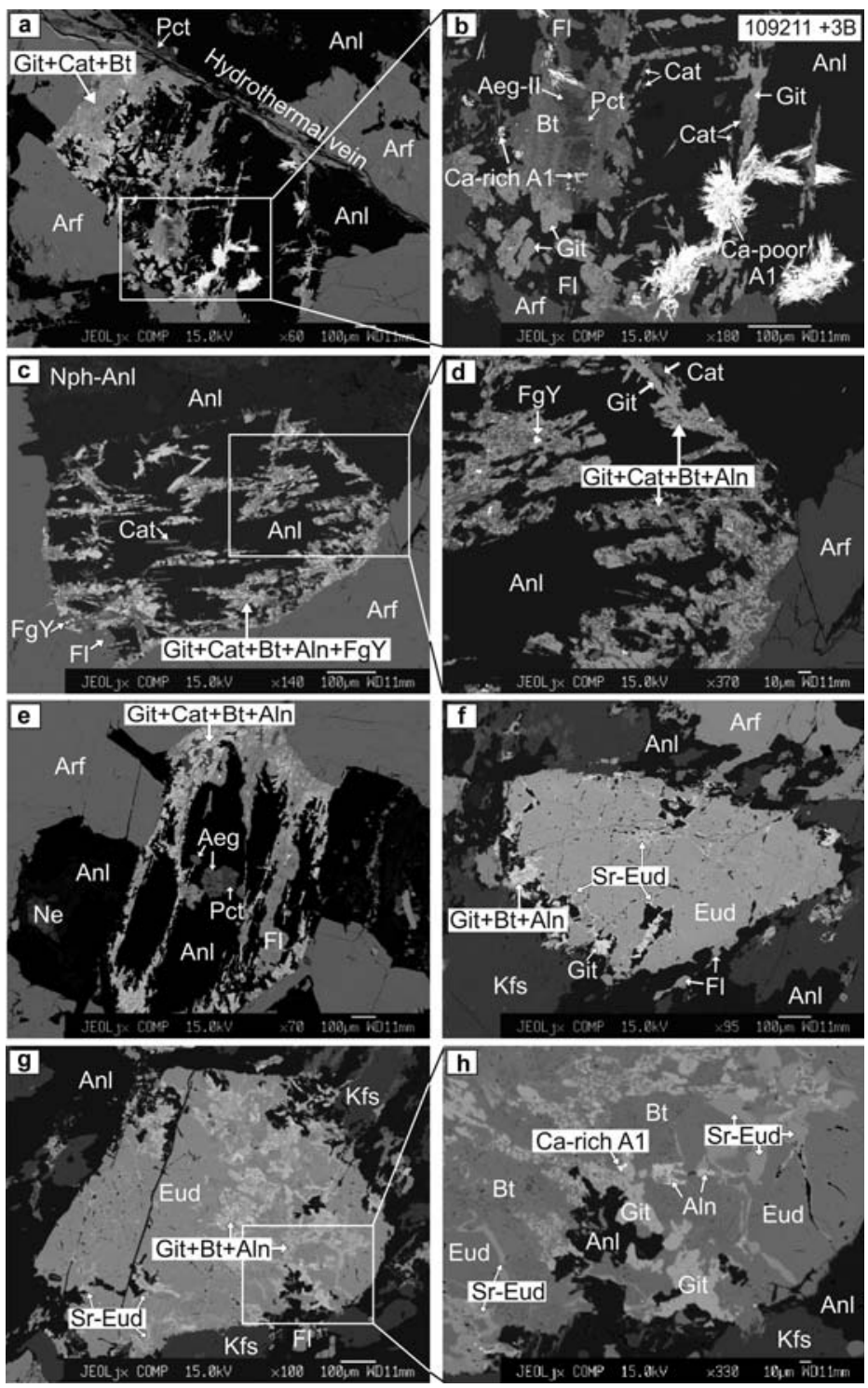

FIG. 5. Gittinsite-bearing (type-II) alteration assemblages after EGM in black kakortokite. Associated alteration minerals include catapleiite (Cat), Sr-rich eudialyte ( $\mathrm{Sr}$-Eud), analcime (Anl), fluorite (Fl), annite (Bt), aegirine (Aeg-II), pectolite (Pct), Ca-rich and Ca-poor A1, allanite-(Ce) (Aln) and fergusonite-(Y) (FgY). (a,b) Type-II pseudomorph after EGM in contact with a hydrothermal vein. Gittinsite is intergrown with annite and catapleiite. Radiating aggregates of Ca-poor A1 crystals, nucleating from gittinsite-catapleiite intergrowths. $(c, d)$ Type-II pseudomorph after EGM showing an overprinted assemblage of gittinsite, catapleiite and annite on former catapleiite laths from type-I alteration paragenesis. Dominant $\mathrm{Nb}$ and $R E E$ phases are fergusonite-(Y) and allanite-(Ce). (e) Gittinsite-bearing pseudomorph after EGM containing intergrowths of gittinsite, catapleiite, allanite-(Ce) and annite, with 'pillars' of fluorite that precipitated around gittinsite-catapleiite intergrowths. $(f, g)$ Remnant EGM crystals with secondary rims of Sr-rich eudialyte and partial decomposition to gittinsite, annite, analcime, allanite-(Ce) and Ca-rich A1. Catapleiite is absent. (h) Detail of partially altered EGM to gittinsite, annite (irregular surface, same shade as EGM), allanite-(Ce) and analcime. The rim of Sr-rich EGM $(5-15 \mu \mathrm{m})$ separates remnants of primary EGM from the gittinsite-annite intergrowths. 
potentially implying a more widespread occurrence of gittinsite in the kakortokites.

\section{Mineral compositions}

\section{Eudialyte-group minerals}

Eudialyte-group minerals belong to large group of $\mathrm{Na}-\mathrm{Ca}-\mathrm{Zr}$ silicates with complex crystallographic structures. The IMA-accepted formula (Johnsen et al., 2003) for $\mathrm{EGM}$ is $\mathrm{N}_{15-16} \mathrm{M}(1)_{6} \mathrm{M}(2)_{3} \mathrm{Z}_{3} \mathrm{M}(3)$ $\mathrm{M}(4) \mathrm{Si}_{24} \mathrm{O}_{66-73}(\mathrm{OH})_{0-9} \mathrm{X}_{2}$, where $\mathrm{N}=\mathrm{Na}^{+}, \mathrm{K}^{+}, \mathrm{Sr}^{2+}$, $\mathrm{Ca}^{2+}, R E E^{3+}, \square, \mathrm{Ba}^{2+}, \mathrm{Mn}^{2+}, \mathrm{H}_{3} \mathrm{O}^{+} ; \mathrm{M}(1)=\mathrm{Ca}^{2+}$, $R E E^{3+}, \mathrm{Mn}^{2+}, \mathrm{Fe}^{2+}, \mathrm{Na}^{+}, \mathrm{Sr}^{2+} ; \mathrm{M}(2)=\mathrm{Fe}^{2+}, \mathrm{Mn}^{2+}$, $\mathrm{Na}^{+}, \square, \mathrm{H}_{3} \mathrm{O}^{+}, \mathrm{Zr}^{4+}, \mathrm{Ta}^{5+}, \mathrm{Ti}^{4+}, \mathrm{K}^{+}, \mathrm{Ba}^{2+} ; \mathrm{M}(3,4)$ $=\mathrm{Si}^{4+}, \mathrm{Al}^{3+}, \mathrm{Nb}^{5+}, \mathrm{Ti}^{4+}, \mathrm{W}^{6+}, \mathrm{Na}^{+} ; \mathrm{Z}=\mathrm{Zr}^{4+}, \mathrm{Hf}^{4+}$, $\mathrm{Ti}^{4+}, \mathrm{Nb}^{5+} ; \mathrm{OH}=\mathrm{H}_{2} \mathrm{O}, \mathrm{OH}^{-}, \mathrm{O}^{2-}, \mathrm{CO}_{3}^{2-}, \mathrm{SO}_{4}^{2-}$, $\mathrm{SiO}_{4}^{4-} ; \mathrm{X}=\mathrm{Cl}^{-}, \mathrm{F}^{-}, \mathrm{OH}^{-}$.

Throughout the kakortokite sequence EGM compositions are relatively constant, closely approaching compositions of eudialyte sensu stricto: $\quad \mathrm{Na}_{15} \mathrm{Ca}_{6} \mathrm{Fe}_{3} \mathrm{Zr}_{3} \mathrm{Si}\left(\mathrm{Si}_{25} \mathrm{O}_{72}\right)\left(\mathrm{O}, \mathrm{OH}, \mathrm{H}_{2} \mathrm{O}\right)_{3}$ $(\mathrm{Cl}, \mathrm{OH})_{2}$, with minor deviations towards $\mathrm{Mn}$-, $R E E$ - and $\mathrm{Nb}$-bearing end-members (kentbrooksite component; Johnsen et al., 2003; Pfaff et al, 2008). Both within individual kakortokite units (Lindhuber et al., 2015), and across the overall stratigraphy (Pfaff et al., 2008), EGM exhibit decreasing molar $\mathrm{Fe} / \mathrm{Mn}$ ratios, interpreted to indicate continued magmatic fractionation (e.g. Schilling et al., 2011; Marks and Mark1, 2015). From the base of the kakortokites towards the lujavrites, average EGM furthermore show decreasing $\mathrm{Ca}$ (6-5 a.p.f.u.), $\mathrm{Cl}(1.5-1$ a.p.f.u.), $\mathrm{Nb}(0.23-$ 0.17 a.p.f.u $)$ and $\mathrm{Hf}$ contents $(0.4-0.3$ a.p.f.u.) and increasing $R E E+\mathrm{Y}(0.45-0.5$ a.p.f.u., Table 1$)$.

Relics of primary EGM in partially replaced grains show little to no compositional modifications relative to unaltered EGM, except for occasional and minor loss of $\mathrm{Na}$ and $\mathrm{Cl}$. This includes preservation of the primary sector zoning in the relict EGM domains. Metasomatic changes leading to distinct secondary EGM compositions have only been observed in the gittinsite-bearing assemblages from layer +3 black, where remnants of primary EGM are mantled by a thin rim $(1-5 \mu \mathrm{m})$ of a Sr-rich and Na-poor hydrothermal EGM variety (taseqite component; Johnsen et al., 2003, Fig. 5f,g,h).

\section{Catapleiite-group minerals}

Catapleiite compositions range within the calciocatapleiite-catapleiite solid solution series
(Table 2), with $\mathrm{Ca}$ contents ranging from 0.2 to 6.3 wt. $\%$ (0.02-0.47 a.p.f.u.) and $\mathrm{Na}$ contents from 7.8 to 14.3 wt. $\%$ (1-1.8 a.p.f.u.). The most Ca-rich catapleiite co-exists with gittinsite in sample 109211. In some pseudomorphs, larger catapleiite crystals $(>30 \mu \mathrm{m})$ are zoned with rims showing minor $\mathrm{Ca}$ enrichment relative to the core ( +0.05 a.p.f.u.). Contents of $\mathrm{Y}_{2} \mathrm{O}_{3}$ and $\mathrm{Hf}_{2} \mathrm{O}$ range between $0.1-0.9$ wt.\% and $0.4-0.7$ wt.\%, respectively, and are weakly inversely correlated with Y increasing and Hf decreasing across the sequence.

\section{Gittinsite}

Gittinsite exhibits no significant zoning or compositional variations. Average gittinsite compositions are in accordance with the ideal formula $\mathrm{CaZrSi}_{2} \mathrm{O}_{7}$ (Table 3). Calcium contents are near-constant (18-19 wt. $\% \mathrm{CaO})$ and the $\mathrm{Na}$ content is generally negligible $\left(<0.1\right.$ wt.\% $\left.\mathrm{Na}_{2} \mathrm{O}\right)$. Contents of $\mathrm{Nb}_{2} \mathrm{O}_{5}, \mathrm{Y}_{2} \mathrm{O}_{3}$ and $\mathrm{Hf}_{2} \mathrm{O}$ range between $0-0.4$ wt. $\%, 0.1-0.3$ wt. $\%$ and $\sim 0.8$ wt. $\%$, respectively.

\section{Clinopyroxene}

Clinopyroxene in the kakortokites and lujavrites are compared to pyroxene from earlier Ilimaussaq rock types (e.g. augite syenites, Larsen, 1976; Markl et al., 2001, Marks and Markl, 2015). At least two generations of pyroxenes are distinguished in most samples: (1) primary magmatic aegirine; and (2) secondary aegirine replacing primary $\mathrm{Fe}$ phases such as arfvedsonite, EGM and aenigmatite. In all EGM pseudomorphs, secondary aegirine forms the main Fe-bearing phase. Additionally, aegirine typically replaces primary arfvedsonite along contacts with altered EGM (Fig. $3 e, f$ ). Magmatic and secondary aegirine can be distinguished petrographically as they exhibit vivid green and brownish green pleochroism, respectively. Primary aegirine exhibits solid-solution trends in the aegirine-augite series with variable $\mathrm{Ca}(0.03-$ 0.16 a.p.f.u.) and low $\mathrm{Al}$ (0.05 a.p.f.u., Table 4). In contrast, secondary aegirine is exclusively Na-rich (1 a.p.f.u.), with low $\mathrm{Ca}$ (0.02 a.p.f.u.) and variable Al (0.01-0.18 a.p.f.u.). Following the Morimoto classification (Morimoto, 1998) for Na-pyroxenes (i.e. Quad-jadeite-aegirine, where Quad represents all $\mathrm{Ca}-\mathrm{Fe}-\mathrm{Mg}$ pyroxenes) magmatic aegirine has relatively high Quad-components (up to $12 \mathrm{~mol} . \%$ ), whereas secondary aegirine has higher jadeite contents (up to $18 \mathrm{~mol} \%$, averages in Table 4). Increasing jadeite-components are inferred to 
TABLE 1. Average core compositions of eudialyte-group minerals from selected samples across the sequence.

\begin{tabular}{|c|c|c|c|c|c|c|c|c|c|c|c|c|c|c|}
\hline \multirow{3}{*}{$\begin{array}{l}\text { Sample no. } \\
\text { Layer }\end{array}$} & \multicolumn{2}{|c|}{520701} & \multicolumn{2}{|c|}{109242} & \multicolumn{2}{|c|}{109204} & \multicolumn{2}{|c|}{109211} & \multicolumn{2}{|c|}{109218} & \multicolumn{2}{|c|}{109236} & \multicolumn{2}{|c|}{540247} \\
\hline & \multicolumn{2}{|c|}{-11 white } & \multicolumn{2}{|c|}{-4 black } & \multicolumn{2}{|c|}{0 red } & \multicolumn{2}{|c|}{3 black } & \multicolumn{2}{|c|}{5 white } & \multicolumn{2}{|c|}{13 white } & \multicolumn{2}{|c|}{ E red } \\
\hline & $\begin{array}{c}\text { Average } \\
19\end{array}$ & $2 \sigma$ & $\begin{array}{c}\text { Average } \\
18\end{array}$ & $2 \sigma$ & $\begin{array}{c}\text { Average } \\
13\end{array}$ & $2 \sigma$ & $\begin{array}{c}\text { Average } \\
5\end{array}$ & $2 \sigma$ & $\begin{array}{c}\text { Average } \\
19\end{array}$ & $2 \sigma$ & $\begin{array}{c}\text { Average } \\
10\end{array}$ & $2 \sigma$ & $\begin{array}{c}\text { Average } \\
12\end{array}$ & $2 \sigma$ \\
\hline $\mathrm{Na}_{2} \mathrm{O}$ & 13.95 & 0.32 & 13.69 & 0.23 & 13.57 & 0.31 & 13.10 & 0.11 & 14.40 & 0.17 & 12.25 & 3.16 & 13.01 & 0.60 \\
\hline $\mathrm{Al}_{2} \mathrm{O}_{3}$ & 0.27 & 0.08 & 0.22 & 0.06 & 0.23 & 0.04 & 0.25 & 0.02 & 0.25 & 0.04 & 0.31 & 0.20 & 0.31 & 0.21 \\
\hline $\mathrm{SiO}_{2}$ & 48.15 & 0.75 & 48.06 & 0.64 & 48.19 & 0.89 & 48.85 & 0.26 & 48.34 & 0.72 & 49.28 & 1.05 & 49.06 & 0.67 \\
\hline $\mathrm{Cl}$ & 1.60 & 0.05 & 1.57 & 0.05 & 1.52 & 0.05 & 1.50 & 0.03 & 1.47 & 0.05 & 1.41 & 0.08 & 1.27 & 0.07 \\
\hline $\mathrm{K}_{2} \mathrm{O}$ & 0.28 & 0.05 & 0.27 & 0.03 & 0.23 & 0.03 & 0.27 & 0.03 & 0.32 & 0.03 & 0.28 & 0.07 & 0.24 & 0.04 \\
\hline $\mathrm{CaO}$ & 10.59 & 0.30 & 10.49 & 0.39 & 10.17 & 0.38 & 10.55 & 0.39 & 10.31 & 0.28 & 9.90 & 0.37 & 9.43 & 0.44 \\
\hline $\mathrm{FeO}$ & 6.20 & 0.26 & 7.09 & 0.17 & 6.31 & 0.22 & 6.32 & 0.23 & 6.12 & 0.16 & 5.54 & 0.19 & 6.37 & 0.39 \\
\hline $\mathrm{MnO}$ & 0.77 & 0.07 & 0.57 & 0.05 & 0.75 & 0.05 & 0.65 & 0.13 & 0.73 & 0.11 & 0.80 & 0.05 & 0.92 & 0.08 \\
\hline $\mathrm{Y}_{2} \mathrm{O}_{3}$ & 0.41 & 0.04 & 0.43 & 0.08 & 0.44 & 0.07 & 0.43 & 0.05 & 0.44 & 0.06 & 0.46 & 0.04 & 0.53 & 0.06 \\
\hline $\mathrm{ZrO}_{2}$ & 11.37 & 0.17 & 11.46 & 0.23 & 11.23 & 0.19 & 10.55 & 0.31 & 10.91 & 0.27 & 10.91 & 0.28 & 10.83 & 0.38 \\
\hline $\mathrm{Nb}_{2} \mathrm{O}_{5}$ & 0.97 & 0.30 & 0.90 & 0.29 & 0.97 & 0.28 & 0.90 & 0.35 & 0.82 & 0.31 & 0.70 & 0.26 & 0.69 & 0.34 \\
\hline $\mathrm{La}_{2} \mathrm{O}_{3}$ & 0.46 & 0.08 & 0.48 & 0.09 & 0.50 & 0.06 & 0.51 & 0.04 & 0.47 & 0.12 & 0.49 & 0.06 & 0.46 & 0.11 \\
\hline $\mathrm{Ce}_{2} \mathrm{O}_{3}$ & 0.90 & 0.16 & 0.89 & 0.14 & 0.95 & 0.12 & 1.06 & 0.11 & 0.93 & 0.24 & 0.95 & 0.11 & 0.97 & 0.16 \\
\hline $\mathrm{Nd}_{2} \mathrm{O}_{3}$ & 0.34 & 0.14 & 0.34 & 0.08 & 0.37 & 0.16 & 0.36 & 0.15 & 0.39 & 0.15 & 0.38 & 0.08 & 0.42 & 0.11 \\
\hline $\mathrm{HfO}_{2}$ & 0.25 & 0.04 & 0.24 & 0.05 & 0.25 & 0.04 & 0.24 & 0.05 & 0.25 & 0.05 & 0.24 & 0.06 & 0.21 & 0.07 \\
\hline $\mathrm{TiO}_{2}$ & 0.07 & 0.02 & 0.06 & 0.02 & 0.06 & 0.04 & 0.05 & 0.02 & 0.05 & 0.02 & 0.04 & 0.03 & 0.05 & 0.02 \\
\hline $\mathrm{O}=\mathrm{Cl}$ & 0.36 & & 0.36 & & 0.34 & & 0.34 & & 0.33 & & 0.32 & & 0.29 & \\
\hline Total & 96.23 & & 96.40 & & 95.38 & & 95.25 & & 95.87 & & 93.63 & & 94.51 & \\
\hline \multicolumn{15}{|c|}{ Formulae based on $(\mathrm{Si}, \mathrm{Zr}, \mathrm{Ti}, \mathrm{Nb}, \mathrm{Al}, \mathrm{Hf})=29$} \\
\hline $\mathrm{Na}$ & 14.38 & & 14.14 & & 14.00 & & 13.44 & & 14.88 & & 12.44 & & 13.27 & \\
\hline $\mathrm{Al}$ & 0.17 & & 0.14 & & 0.15 & & 0.16 & & 0.16 & & 0.19 & & 0.20 & \\
\hline $\mathrm{Si}$ & 25.59 & & 25.61 & & 25.65 & & 25.85 & & 25.75 & & 25.80 & & 25.81 & \\
\hline $\mathrm{Cl}$ & 1.44 & & 1.42 & & 1.37 & & 1.34 & & 1.33 & & 1.25 & & 1.14 & \\
\hline $\mathrm{K}$ & 0.19 & & 0.19 & & 0.16 & & 0.18 & & 0.21 & & 0.19 & & 0.16 & \\
\hline $\mathrm{Ca}$ & 6.03 & & 5.99 & & 5.80 & & 5.98 & & 5.89 & & 5.56 & & 5.32 & \\
\hline $\mathrm{Fe}$ & 2.76 & & 3.16 & & 2.81 & & 2.80 & & 2.73 & & 2.42 & & 2.80 & \\
\hline $\mathrm{Mn}$ & 0.35 & & 0.26 & & 0.34 & & 0.29 & & 0.33 & & 0.35 & & 0.41 & \\
\hline Y & 0.12 & & 0.12 & & 0.12 & & 0.12 & & 0.13 & & 0.13 & & 0.15 & \\
\hline $\mathrm{Zr}$ & 2.95 & & 2.98 & & 2.91 & & 2.72 & & 2.84 & & 2.79 & & 2.78 & \\
\hline
\end{tabular}




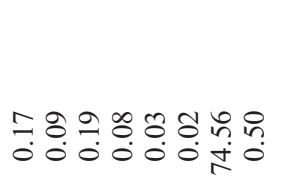

reflect decreasing silica activity with progressive crystallization and differentiation (Markl et al., 2001), and as such fit a late-stage/hydrothermal origin. Zirconium contents range from 0 to $1.6 \mathrm{wt} . \%$ in magmatic aegirine, vs. 0 to 2.7 wt. $\%$ in secondary aegirine.
\end{abstract}

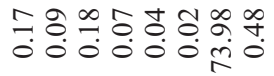

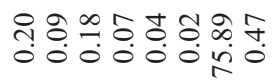

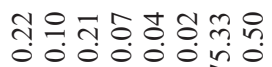

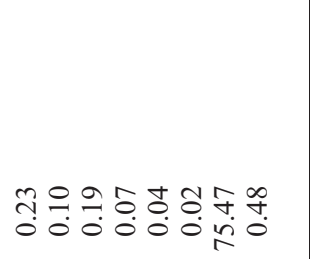

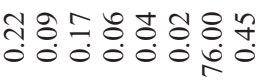

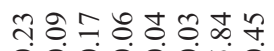

000000 in

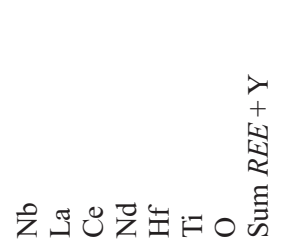

\section{Nacareniobsite-(Ce) and rinkite-group minerals}

Nacareniobsite-(Ce) $\left(\mathrm{Na}_{3} \mathrm{Ca}_{3} R E E \mathrm{Nb}\left(\mathrm{Si}_{2} \mathrm{O}_{7}\right)_{2} \mathrm{OF}_{3}\right)$ is common in most EGM pseudomorph assemblages and contains $\sim 14$ wt. $\%$ light rare-earth oxides (LREO; $\mathrm{Ce}+\mathrm{La}+\mathrm{Nd}$ ) and $\sim 15$ wt. $\% \mathrm{Nb}_{2} \mathrm{O}_{5}$. In the absence of fluorite or pectolite, nacareniobsite(Ce) incorporates most of the $\mathrm{Ca}$ released from EGM. Nacareniobsite-(Ce) occurs either as elongate prismatic crystals $(<30 \mu \mathrm{m})$ or interstitially to catapleiite laths (Fig. $3 c, g, h$ ). Sector zoning was observed in some crystals. Nacareniobsite-(Ce) is related closely to the rinkite-mosandrite group minerals (ideally $\left.\mathrm{Na}_{2}(\mathrm{Ca}, R E E)_{4} \mathrm{Ti}\left(\mathrm{Si}_{2} \mathrm{O}_{7}\right)_{2} \mathrm{OF}_{3}\right)$, and represents the $\mathrm{Nb}$-rich analogue following the coupled substitution $\mathrm{Nb}^{5+}+\mathrm{Na}^{+} \leftrightarrow \mathrm{Ti}^{4+}+\mathrm{Ca}^{2+}$ (e.g. Sokolova and Hawthorne, 2008; Cámara et al., 2011; Rønsbo et al., 2013). Both rinkite and nacareniobsite-(Ce) were first described from Ilímaussaq (Lorenzen, 1884; Petersen et al., 1989). Rinkite is an accessory intercumulus mineral and is relatively abundant in white kakortokites. In contrast to magmatic rinkite, which shows significant solid solution between $\mathrm{Ti}$ and $\mathrm{Nb}$ and has low $\mathrm{Na}, \mathrm{Ca}$ and F contents (Rønsbo et al., 2013), secondary nacareniobsite-(Ce) shows near ideal end-member compositions. In common with the EGM, magmatic rinkite has been subjected to significant late-stage alteration (Rønsbo et al., 2013).

\section{Other REE phases}

Besides nacareniobsite-(Ce) the most common $R E E$ minerals in the alteration assemblages are Ca-rich and $\mathrm{Ca}$-poor varieties of the $R E E$ silicates A1 (Karup-Møller and Rose-Hansen 2013; Fig. 3c, $e, g, h)$. These generally exhibit acicular or platy habits and are typically smaller than $5 \mu \mathrm{m}$. Semi-quantitative EDS analyses suggest that A1 minerals contain up to 65 wt.\% LREO and thus represent a significant host for the released $R E E$. Our analyses also indicate the presence of some varieties that are richer in $\mathrm{P}$ and poorer in $R E E$, approaching compositions of britholite-(Ce) (following a $R E E^{3+}+\mathrm{Si}^{4+} \Leftrightarrow \mathrm{Ca}^{2+}+\mathrm{P}^{5+}$ coupled 
TABLE 2. Average compositions of catapleiite-group minerals from selected samples across the sequence.

\begin{tabular}{|c|c|c|c|c|c|c|c|c|c|c|c|c|c|c|c|c|c|c|c|c|}
\hline \multirow{3}{*}{$\begin{array}{l}\text { Sample no. } \\
\text { Layer }\end{array}$} & \multicolumn{2}{|c|}{520704} & \multicolumn{2}{|c|}{109255} & \multicolumn{2}{|c|}{109208} & \multicolumn{2}{|c|}{109207} & \multicolumn{2}{|c|}{109211} & \multicolumn{2}{|c|}{109212} & \multicolumn{2}{|c|}{109213} & \multicolumn{2}{|c|}{109229} & \multicolumn{2}{|c|}{540247} & \multicolumn{2}{|c|}{540279} \\
\hline & \multicolumn{2}{|c|}{-10 white } & \multicolumn{2}{|c|}{-6 white } & \multicolumn{2}{|c|}{1 white } & \multicolumn{2}{|c|}{$1 \mathrm{red}$} & \multicolumn{2}{|c|}{3 black } & \multicolumn{2}{|c|}{3 red } & \multicolumn{2}{|c|}{3 white } & \multicolumn{2}{|c|}{$10 \mathrm{red}$} & \multicolumn{2}{|c|}{ E red } & \multicolumn{2}{|c|}{ Aeg Lj I } \\
\hline & $\begin{array}{c}\text { Average } \\
6\end{array}$ & $2 \sigma$ & $\begin{array}{c}\text { Average } \\
12\end{array}$ & $2 \sigma$ & $\begin{array}{c}\text { Average } \\
6\end{array}$ & $2 \sigma$ & $\begin{array}{c}\text { Average } \\
11\end{array}$ & $2 \sigma$ & $\begin{array}{c}\text { Average } \\
2\end{array}$ & $2 \sigma$ & $\begin{array}{c}\text { Average } \\
19\end{array}$ & $2 \sigma$ & $\begin{array}{c}\text { Average } \\
9\end{array}$ & $2 \sigma$ & $\begin{array}{c}\text { Average } \\
13\end{array}$ & $2 \sigma$ & $\begin{array}{c}\text { Average } \\
12\end{array}$ & $2 \sigma$ & $\begin{array}{c}\text { Average } \\
5\end{array}$ & $2 \sigma$ \\
\hline $\mathrm{Na}_{2} \mathrm{O}$ & 13.65 & 2.04 & 13.76 & 0.85 & 8.04 & 0.78 & 8.88 & 1.70 & 8.25 & 0.19 & 8.21 & 1.16 & 10.61 & 2.19 & 9.35 & 2.84 & 13.30 & 1.01 & 9.24 & 1.05 \\
\hline $\mathrm{SiO}_{2}$ & 43.52 & 0.43 & 43.44 & 0.51 & 43.05 & 0.99 & 43.20 & 1.32 & 42.39 & 0.87 & 43.27 & 0.35 & 43.60 & 0.90 & 43.25 & 1.18 & 43.40 & 0.80 & 43.82 & 0.58 \\
\hline $\mathrm{CaO}$ & 0.24 & 0.26 & 0.23 & 0.34 & 5.80 & 1.08 & 4.50 & 1.82 & 6.31 & 1.99 & 5.80 & 1.38 & 1.28 & 1.37 & 3.86 & 3.72 & 0.38 & 0.43 & 3.12 & 1.38 \\
\hline $\mathrm{FeO}$ & 0.16 & 0.19 & 0.05 & 0.14 & 0.08 & 0.12 & 0.19 & 0.10 & 0.25 & 0.40 & 0.05 & 0.08 & 0.14 & 0.35 & 0.27 & 1.32 & 0.29 & 0.31 & 0.09 & 0.06 \\
\hline $\mathrm{MnO}$ & 0.01 & 0.04 & 0.00 & 0.05 & 0.01 & 0.04 & 0.03 & 0.12 & 0.01 & 0.06 & 0.01 & 0.03 & 0.01 & 0.04 & 0.11 & 0.30 & 0.06 & 0.15 & 0.04 & 0.04 \\
\hline $\mathrm{Y}_{2} \mathrm{O}_{3}$ & 0.26 & 0.62 & 0.15 & 0.58 & 0.32 & 0.40 & 0.18 & 0.55 & 0.37 & 0.11 & 0.14 & 0.20 & 0.29 & 0.53 & 0.57 & 0.61 & 0.42 & 0.48 & 0.66 & 0.71 \\
\hline $\mathrm{ZrO}_{2}$ & 29.81 & 1.93 & 30.78 & 0.96 & 29.46 & 1.00 & 30.30 & 2.04 & 29.78 & 0.88 & 30.12 & 0.69 & 30.31 & 1.85 & 29.10 & 2.40 & 30.12 & 1.66 & 30.20 & 1.50 \\
\hline $\mathrm{Nb}_{2} \mathrm{O}_{5}$ & 0.50 & 1.96 & b.d.* & & 0.10 & 0.38 & b.d. & & 0.18 & 0.26 & b.d. & & 0.35 & 1.05 & 0.96 & 0.96 & 0.19 & 0.29 & 0.20 & 0.38 \\
\hline $\mathrm{HfO}_{2}$ & 0.62 & 0.13 & 0.68 & 0.15 & 0.60 & 0.15 & 0.69 & 0.14 & 0.61 & 0.22 & 0.67 & 0.08 & 0.67 & 0.16 & 0.59 & 0.16 & 0.55 & 0.08 & 0.44 & 0.14 \\
\hline Total** & 88.80 & & 89.09 & & 87.47 & & 87.96 & & 88.15 & & 88.28 & & 87.26 & & 88.07 & & 88.71 & & 87.79 & \\
\hline \multicolumn{21}{|c|}{ Fomulae based on 9 oxygens } \\
\hline $\mathrm{Na}$ & 1.82 & & 1.83 & & 1.08 & & 1.19 & & 1.11 & & 1.10 & & 1.43 & & 1.25 & & 1.78 & & 1.23 & \\
\hline $\mathrm{Si}$ & 2.99 & & 2.99 & & 2.99 & & 2.99 & & 2.94 & & 2.98 & & 3.03 & & 2.98 & & 2.99 & & 3.02 & \\
\hline $\mathrm{Ca}$ & 0.02 & & 0.02 & & 0.43 & & 0.33 & & 0.47 & & 0.43 & & 0.10 & & 0.29 & & 0.03 & & 0.23 & \\
\hline $\mathrm{Fe}$ & 0.01 & & 0.00 & & 0.00 & & 0.01 & & 0.01 & & 0.00 & & 0.01 & & 0.02 & & 0.02 & & 0.00 & \\
\hline $\mathrm{Mn}$ & 0.00 & & 0.00 & & 0.00 & & 0.00 & & 0.00 & & 0.00 & & 0.00 & & 0.01 & & 0.00 & & 0.00 & \\
\hline Y & 0.01 & & 0.01 & & 0.01 & & 0.01 & & 0.01 & & 0.01 & & 0.01 & & 0.02 & & 0.02 & & 0.02 & \\
\hline $\mathrm{Zr}$ & 1.00 & & 1.03 & & 1.00 & & 1.02 & & 1.01 & & 1.01 & & 1.03 & & 0.98 & & 1.01 & & 1.01 & \\
\hline $\mathrm{Nb}$ & 0.02 & & 0.00 & & 0.00 & & 0.00 & & 0.01 & & 0.00 & & 0.01 & & 0.03 & & 0.01 & & 0.01 & \\
\hline Hf & 0.01 & & 0.01 & & 0.01 & & 0.01 & & 0.01 & & 0.01 & & 0.01 & & 0.01 & & 0.01 & & 0.01 & \\
\hline
\end{tabular}

*b.d. = below detection limit.

** Low totals due to high water contents. 
TABLE 3. Average composition of gittinsite.

\begin{tabular}{lcccc}
\hline Sample no. & \multicolumn{4}{c}{109211 (3 black) } \\
\cline { 2 - 5 }$n$ & $\begin{array}{c}\text { Average* } \\
n\end{array}$ & $2 \sigma$ & $\begin{array}{c}\text { Average** } \\
7\end{array}$ & $2 \sigma$ \\
\hline $\mathrm{Na}_{2} \mathrm{O}$ & 0.10 & 0.29 & b.d & \\
$\mathrm{SiO}_{2}$ & 39.02 & 0.45 & 41.19 & 0.25 \\
$\mathrm{CaO}$ & 18.29 & 0.55 & 19.10 & 0.73 \\
$\mathrm{ZrO}_{2}$ & 38.49 & 1.54 & 39.80 & 1.18 \\
$\mathrm{Nb}_{2} \mathrm{O}_{5}$ & 0.16 & 0.72 & 0.39 & 0.62 \\
$\mathrm{HfO}_{2}$ & 0.78 & 0.12 & n.a. & \\
Total & 96.84 & & 100.47 &
\end{tabular}

Fomulae based on 7 oxygens

$\begin{array}{lcc}\mathrm{Na} & 0.01 & - \\ \mathrm{Si} & 2.00 & 2.02 \\ \mathrm{Ca} & 1.01 & 1.01 \\ \mathrm{Zr} & 0.96 & 0.95 \\ \mathrm{Nb} & 0.00 & 0.01 \\ \mathrm{Hf} & 0.01 & -\end{array}$

*Cameca analyses at the University of Oslo.

$* * \mathrm{JEOL}$ analyses at the University of Copenhagen.

substitution scheme; cf. Karup-Møller et al., 2010; A1 with apatite structure). An unknown Ba-rich REE silicate (c. 6-10 wt.\% $\mathrm{BaO}, 0-1 \%$ $\mathrm{CaO}, 2 \% \mathrm{Nb}_{2} \mathrm{O}_{5}, 55 \%$ LREO, $20 \% \mathrm{SiO}_{2}$ and $2 \% \mathrm{~F}$ ) was observed in six samples. These minerals exhibit similar molar proportions to the A1 REE silicates, with inversely correlated $\mathrm{Ca}$ and $\mathrm{Ba}$ contents. The Ba-REE silicates have similar habits (radiating fibrous or platy) and back-scatter index as the Al minerals, making them indistinguishable without compositional data. Several of the above-mentioned REE phases can occur together in a single pseudomorph. Catapleiite-type pseudomorphs containing secondary apatite and fluorite contain monazite as the main REE phase (Fig. 4). Monazite was only observed in white kakortokites. The gittinsite-bearing assemblages contain allanite-(Ce), as well as Ca-poor A1 minerals, fergusonite-(Y) and an unidentified $\mathrm{Ca}-H R E E-Y$ silicate.

\section{Discussion}

\section{EGM alteration; characterization of assemblages and mechanisms}

Petrographic observations of the kakortokites at Ilímaussaq demonstrate substantial subsolidus alteration of primary minerals including decomposition of the primary HFSE phases EGM and rinkite, zeolitization of primary feldspars and feldspathoids and alteration of Fe phases arfvedsonite and aenigmatite. Three types of EGM decomposition assemblages are distinguished based on the presence of the secondary zirconosilicates catapleiite, zircon or gittinsite (Fig. 6). The gittinsite-bearing paragenesis (type-II, Fig. 6) represents a previously unidentified EGM alteration assemblage and was found to be pervasive in one sample and rarely dispersed in another, in both cases overprinting a previous catapleiite-alteration paragenesis.

Catapleiite-bearing assemblages (type-I, Fig. 6) are dominant throughout the kakortokites and most agpaitic units of Ilímaussaq. Zircon-bearing assemblages (type-III, Fig. 6) were not observed in the current study, which confirms their restricted occurrence to the more extensively altered kakortokites, lujavrites and pegmatites (Karup-Møller et al., 2010). Alteration of EGM to catapleiite is commonly explained by interaction with hydrous interstitial melts, or late-magmatic aqueous fluids at the final stages of crystallization (Ussing, 1912; KarupMøller et al., 2010; Karup-Møller and RoseHansen, 2013). The existence of such late-stage fluids is confirmed by the presence of aqueous, commonly highly saline, fluid inclusions in secondary phases in the kakortokites (Konnerup-Madsen, 2001). In contrast, zircon-bearing assemblages were inferred to result from fluids expelled by late lujavrite melts or from external fluids with reduced alkalinity (Karup-Møller et al., 2010). Both catapleiite- and zircon-bearing EGM alteration assemblages have also been described from several other agpaitic complexes, i.e. North Qôroq (Greenland), Pilanesberg (South Africa), Sushina Hill (India) and Tamazeght (Morocco) (e.g. Coulson, 1997; Khadem Allah et al., 1998; Mitchell and Liferovich, 2006; Mitchell and Chakrabarty, 2012; Chakrabarty et al., 2012; 2013). Other $\mathrm{Zr}$ phases reported from EGM alteration assemblages include hilairite $\left(\mathrm{Na}_{2} \mathrm{ZrSi}_{3} \mathrm{O}_{9} \cdot 3 \mathrm{H}_{2} \mathrm{O}\right)$ and vlasovite $\left(\mathrm{Na}_{2} \mathrm{ZrSi}_{4} \mathrm{O}_{11}\right)$, from Tamazeght and Pilanesberg, respectively (Khadem Allah et al., 1998; Mitchell and Liferovich, 2006).

Zirconosilicate assemblages are typically characterized as agpaitic (containing complex alkalizirconosilicates, e.g. catapleiite/gaiddonayite, hilairite or vlasovite) or miaskitic (containing simple zirconosilicates, e.g. zircon or baddelyite). Allanite-(Ce) was found in association with zirconbearing assemblages, both at Ilímaussaq (KarupMøller et al., 2010) and other localities (e.g. 
A. M. BORST ETAL.

TABLE 4. Average compositions of magmatic and secondary aegirine.

\begin{tabular}{|c|c|c|c|c|}
\hline \multirow{2}{*}{$\begin{array}{l}\text { Mineral } \\
n\end{array}$} & \multicolumn{2}{|c|}{ Primary aegirine } & \multicolumn{2}{|c|}{ Secondary aegirine } \\
\hline & $\begin{array}{c}\text { Average } \\
102\end{array}$ & $2 \sigma$ & $\begin{array}{c}\text { Average } \\
54\end{array}$ & $2 \sigma$ \\
\hline $\mathrm{Na}_{2} \mathrm{O}$ & 13.28 & 0.85 & 14.52 & 0.78 \\
\hline $\mathrm{SiO}_{2}$ & 52.70 & 2.49 & 53.45 & 1.86 \\
\hline $\mathrm{Al}_{2} \mathrm{O}_{3}$ & 1.18 & 0.28 & 1.97 & 1.89 \\
\hline $\mathrm{FeO}$ & 29.02 & 2.09 & 27.66 & 2.82 \\
\hline $\mathrm{MgO}$ & 0.13 & 0.13 & 0.09 & 0.15 \\
\hline $\mathrm{MnO}$ & 0.15 & 0.12 & 0.21 & 0.33 \\
\hline $\mathrm{CaO}$ & 2.61 & 1.24 & 0.47 & 1.19 \\
\hline $\mathrm{K}_{2} \mathrm{O}$ & 0.01 & 0.02 & 0.02 & 0.11 \\
\hline $\mathrm{TiO}_{2}$ & 0.72 & 0.71 & 0.60 & 0.92 \\
\hline $\mathrm{ZrO}_{2}$ & 0.31 & 0.37 & 0.68 & 1.43 \\
\hline Total & 100.11 & & 99.69 & \\
\hline \multicolumn{5}{|c|}{ Fomulae based on 4 cations } \\
\hline $\mathrm{Na}$ & 0.95 & & 1.04 & \\
\hline $\mathrm{Si}$ & 1.95 & & 1.97 & \\
\hline $\mathrm{Al}$ & 0.05 & & 0.09 & \\
\hline $\mathrm{Fe}^{3+} *$ & 0.85 & & 0.85 & \\
\hline $\mathrm{Fe}^{2+}$ & 0.05 & & 0.00 & \\
\hline $\mathrm{Mg}$ & 0.01 & & 0.01 & \\
\hline $\mathrm{Mn}$ & 0.00 & & 0.01 & \\
\hline $\mathrm{Ca}$ & 0.10 & & 0.02 & \\
\hline $\mathrm{K}$ & 0.00 & & 0.00 & \\
\hline $\mathrm{Ti}$ & 0.02 & & 0.02 & \\
\hline $\mathrm{Zr}$ & 0.01 & & 0.01 & \\
\hline $\mathrm{O}$ & 6.00 & & 5.95 & \\
\hline \multicolumn{5}{|c|}{ Morimoto end-members** } \\
\hline Quad & 5.49 & & 1.13 & \\
\hline Jadeite & 1.65 & & 8.37 & \\
\hline Aegirine & 92.87 & & 90.50 & \\
\hline
\end{tabular}

* $\mathrm{Fe}^{3+}$ and $\mathrm{Fe}^{2+}$ recalculated to 6 oxygens.

**Based on Morimoto (1988).

Coulson, 1997; Khadem-Allah et al., 1998; Mitchell and Liferovich, 2006), but not with agpaitic alteration assemblages and is therefore characterized as miaskitic. With allanite-(Ce) being part of the gittinsite-bearing paragenesis, the latter can be regarded as transitional-miaskitic (Fig. 6).

Several stages of alteration can lead to multiple generations of superimposed agpaitic and miaskitic assemblages (Mitchell and Liferovich, 2006). The relative stability of the zirconosilicates depends on parameters such as pressure, temperature and the chemical potential of volatiles and other components in the melts and fluids (e.g. Marr and Wood, 1992; Markl et al., 2001; Mitchell and Liferovich, 2006; Mitchell and Chakrabarty, 2012; Andersen et al., 2010; Marks et al., 2011). As such, EGM decomposition provides a useful tool for monitoring chemical changes in late- and post-magmatic fluids during the late-stage evolution of peralkaline complexes (e.g. Coulson, 1997; Mitchell and Liferovich, 2006; Mitchell and Chakrabarty, 2012). For example, Mitchell and Liferovich (2006) linked the sequence of agpaitic and miaskitic assemblages in the lujavrites of Pilanesberg to changes in the $\mathrm{pH}$, governed by fluctuating $\mathrm{Na} / \mathrm{Cl}$ in response to fluid-rock reactions involving nepheline, sodalite and EGM.

In addition to physico-chemical controls, the proportion and type of secondary phases, particularly those incorporating the released $R E E, \mathrm{Y}, \mathrm{Fe}$, $\mathrm{Mn}, \mathrm{Sr}, \mathrm{Nb}, \mathrm{Hf}$ and $\mathrm{Ti}$, are governed by the primary 


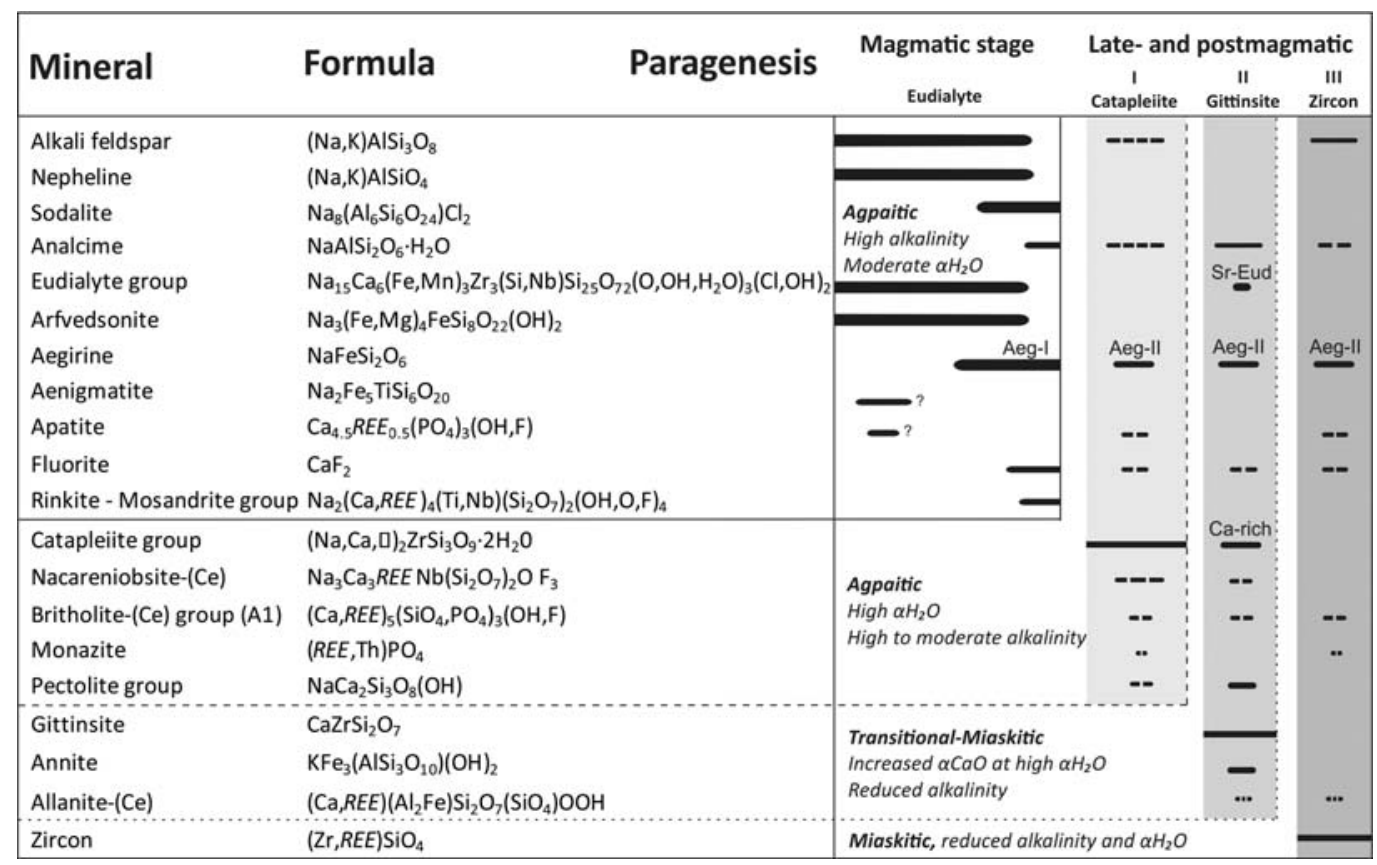

FIG. 6. Generalized paragenetic diagram for the kakortokites and EGM decomposition assemblages. Note that the crystallization sequence at the magmatic stage is slightly different for individual layers of black, red and white kakortokites. EGM alteration parageneses are distinguished based on the $\mathrm{Zr}$ phases catapleiite, gittinsite and zircon, and are characterized, respectively, as agpaitic, transitional and miaskitic (see text for details). Zircon-bearing assemblages (type-III) are obtained from Karup-Møller et al. (2010). Catapleiite-bearing assemblages (type-I) are prevalent throughout the kakortokites, whereas type-II and III are spatially restricted. The gittinsite-bearing assemblage (type-II) is superimposed on a type-I paragenesis. Length and continuation of bars indicate relative abundance and proportions within the pseudomorphs.

composition of the replaced EGM. Given the broad compositional range of EGM worldwide (e.g. Schilling et al., 2011) it is not surprising that the proportion and composition of secondary phases in EGM alteration assemblages may be unique for each locality (see overview Mitchell and Chakrabarty, 2012). For example, EGM at Sushina Hill are particularly Fe-poor and Mn-rich and, accordingly, their alteration assemblages contain the Mn-mineral serandite instead of aegirine (Mitchell and Chakrabarty, 2012). Similarly, REE rich hydrothermal EGM at Pilanesberg produce alteration assemblages with high proportions of secondary $R E E$ phases such as britholite-(Ce) and fergusonite(Y) (Olivo and Williams-Jones, 1999).

As described above, EGM compositions in the kakortokite-lujavrite sequence are relatively constant, except for minor fractionation trends in $\mathrm{Ca}$, $\mathrm{Mn}, \mathrm{Hf}, R E E$ and $\mathrm{Y}$ contents across the stratigraphy (Pfaff et al., 2008; Lindhuber et al., 2015; this work, Table 1). These variations, however, appear too small to cause systematic trends in the composition and type of EGM alteration assemblages across the stratigraphy. The only compositional feature which may be inherited from the precursor EGM is a weakly-defined increase in Y and decrease in $\mathrm{Hf}$ in catapleiite. We infer that most variations in the alteration assemblages reflect different physico-chemical properties of the latestage magmatic/hydrothermal fluids, and as such present an ideal case for modelling of zirconosilicate stabilities.

\section{Chemographic analyses}

Theoretical phase diagrams on the relative stability of catapleiite, zircon and EGM in peralkaline assemblages were presented previously by Andersen et al., 2010 and Marks et al., 2011. 
These show that increasing $\mathrm{H}_{2} \mathrm{O}$ activity, decreased alkalinity (activity of $\mathrm{Na}$ in the form of $\mathrm{Na}_{2} \mathrm{Si}_{2} \mathrm{O}_{5}$, $\mathrm{Na}_{2} \mathrm{SiO}_{3}$ or $\mathrm{NaO}_{2}$ ) and lower $\mathrm{Cl}^{-}$activity favours catapleiite stabilization after EGM, whereas zircon formation requires a further decrease in alkalinity as well as a decrease in $\mathrm{H}_{2} \mathrm{O}$ activity. The relative stability of Ca-rich gittinsite in these mineral assemblages has not been discussed so far.

To further explore the factors governing the observed assemblages in the kakortokites we applied Schreinemakers chemographic analyses to construct semi-quantitative $3 \mathrm{D}$ petrogenetic grids as a function of alkalinity, $\mathrm{H}_{2} \mathrm{O}$ and $\mathrm{CaO}$ activities in the melt/fluid (see methods in Andersen et al., 2010; 2013 and references therein). This was undertaken for a 13-component system including $\mathrm{NaO}_{0.5^{-}}$ $\mathrm{KO}_{0.5}-\mathrm{CaO}-\mathrm{FeO}-R E E \mathrm{O}_{1.5}-\mathrm{ZrO}_{2}-\mathrm{TiO}_{2}-\mathrm{NbO}_{2.5}-$ $\mathrm{AlO}_{1.5}-\mathrm{SiO}_{2}-\mathrm{HO}_{0.5}-\mathrm{FO}_{-0.5}-\mathrm{ClO}_{-0.5}$, combining $\mathrm{Mn}$ and $\mathrm{Mg}$ with $\mathrm{Fe}$, Hf with $\mathrm{Zr}$, and $\mathrm{Y}$ with $R E E$ (Table $5 a$ ). In our diagrams, alkalinity is expressed by the activity of the water-soluble sodium metasilicate component $N m s=\mathrm{Na}_{2} \mathrm{SiO}_{3}$. Divariant reactions were calculated for zirconosilicate assemblages involving EGM, catapleiite, gittinsite, vlasovite and zircon, assuming the co-existing non-HFSE phases analcime, annite, aegirine, pectolite and fluorite (Table 5b). Stoichiometric end-member compositions were used for all phases except catapleiite and EGM, for which we used average compositions (Table $5 a$ ). Calculations (not presented) using the co-existing non-HFSE phases albite, K-feldspar, pectolite and fluorite (type-I and type-III alteration assemblages, Fig. 6) yielded similar configurations, with only minor differences in the slopes of the divariant planes involving catapleiite and EGM.

External buffering was assumed for the components $\mathrm{TiO}_{2}, R E E_{2} \mathrm{O}_{3}$ and $\mathrm{Nb}_{2} \mathrm{O}_{5}$ by phases not included in the analyses (e.g. nacareniobsite-(Ce), fergusonite-(Y), monazite, A1 minerals, etc). The model assumes isobaric-isothermal conditions ( $\sim 1 \mathrm{kbar}$ and $450^{\circ} \mathrm{C}$, Mark1 et al., 2001; Mark1 and Baumgartner, 2002). Pressure-temperature variations influence the relative sizes of the stability volumes, but not the general topology. The effect of oxygen fugacity was disregarded by excluding ferric iron from the calculations, but is known to increase during the final stages of evolution from Fe-mineral equilibria (Markl et al., 2001). Similarly, the effects of $\mathrm{F}$ and $\mathrm{Cl}$ are not considered here but are relevant for the discussion on REE and HFSE mobility; i.e. $\mathrm{F}^{-}$activity is assumed at the fluorite saturation level and a general decrease in $\mathrm{Cl}^{-}$activity is inferred from the fact that all secondary phases are devoid of $\mathrm{Cl}$ (e.g. Andersen et al., 2010; Marks et al., 2011). Finally, Si activity remains well below quartz saturation, corresponding to nepheline-alkali feldspar coexistence.

The calculated reactions were used to construct a grid of divariant planes and univariant lines in 3D $\log a_{\mathrm{Nms}}-\log \mathrm{a}_{\mathrm{H}_{2} \mathrm{O}}-\log a_{\mathrm{CaO}}$ space (Fig. 7). Together, they separate trivariant single-phase stability volumes for EGM, zircon, catapleiite, vlasovite and gittinsite. Vlasovite is neither observed nor reported from the kakortokites, but was included for comparison with the agpaitic type locality of gittinsite at Kipawa, where gittinsite occurs with apophyllite and vlasovite in EGM-rich pegmatites (Ansell, 1980). The resulting petrogenetic grid is internally consistent and gives a true representation of the relative slopes of the divariant planes and univariant lines, and the relative positions of the stability volumes in 3D log activity space. It is semi-quantitative in the sense that no absolute values can be assigned to the axes or the actual sizes of the stability fields without existing quantitative thermodynamic and experimental data. A petrogenetic grid in $\log$ activity space constructed in this way, however, is most useful when predicting the consequences of changes in component activities in melt or fluid. A grid configuration that agrees with the observed mineral assemblages is presented in Fig. 7.

\section{Alteration of EGM to catapleiite}

Eudialyte-group minerals are stable at high levels of alkalinity and variable $\mathrm{H}_{2} \mathrm{O}$ and $\mathrm{CaO}$ activities, whereas catapleiite becomes the dominant zirconosilicate at high $\mathrm{H}_{2} \mathrm{O}$ activity (Fig. 7). The reaction from EGM to catapleiite (reaction 1 in Table $5 b$ ) is represented by the divariant plane between the red and blue volumes at high $a_{\mathrm{H}_{2} \mathrm{O}}$ and $a_{\mathrm{Nms}}$ in Fig. 7 and can be achieved by either: (1) increasing the $a_{\mathrm{H}_{2} \mathrm{O}} ;(2)$ decreasing the alkalinity in a system at high $a_{\mathrm{H}_{2} \mathrm{O}}$; or (3) increasing $a_{\mathrm{CaO}}$ in a system at high $a_{\mathrm{H}_{2} \mathrm{O}}$ and $a_{\mathrm{Nms}}$ (Fig. 7). Assuming that most EGM alteration occurred at relatively low to medium $a_{\mathrm{CaO}}$, increasing water activity appears to be the dominant factor. This can be illustrated in a 2D section perpendicular to the $\log a_{\mathrm{CaO}}$ axis, showing relative stabilities as a function of water activity and alkalinity (Fig. 8). The section is positioned just above the vlasovite field, which is restricted to lower $a_{\mathrm{CaO}}$ and $a_{\mathrm{H}_{2} \mathrm{O}}$. Given the presence of $\mathrm{Ca}$ phases fluorite, apatite and pectolite in both magmatic and post-magmatic assemblages, $a_{\mathrm{CaO}}$ may be too high to allow stabilization of vlasovite in 
TABLE $5 a$. Compositions of minerals and components (in moles per formula unit) used in modelling.

\begin{tabular}{|c|c|c|c|c|c|c|c|c|c|c|c|c|c|c|c|}
\hline & & $\mathrm{NaO}_{0.5}$ & $\mathrm{KO}_{0.5}$ & $\mathrm{CaO}$ & $\mathrm{FeO}$ & $\mathrm{FeO}_{1.5}$ & REE $\mathrm{O}_{1.5}$ & $\mathrm{ZrO}_{2}$ & $\mathrm{TiO}_{2}$ & $\mathrm{NbO}_{2.5}$ & $\mathrm{AlO}_{1.5}$ & $\mathrm{SiO}_{2}$ & $\mathrm{HO}_{0.5}$ & $\mathrm{FO}_{-0.5}$ & $\mathrm{ClO}_{-0.5}$ \\
\hline \multicolumn{16}{|l|}{ Minerals } \\
\hline Eudialyte* & Eud & 14.04 & 0.20 & 5.68 & 2.78 & 0.00 & 0.48 & 2.85 & 0.02 & 0.21 & 0.16 & 25.74 & 2.00 & 0.00 & 1.29 \\
\hline Catapleiite & Cat & 1.20 & 0.00 & 0.40 & 0.00 & 0.00 & 0.00 & 1.00 & 0.00 & 0.00 & 0.00 & 3.00 & 4.00 & 0.00 & 0.00 \\
\hline Gittinsite & Git & 0.00 & 0.00 & 1.00 & 0.00 & 0.00 & 0.00 & 1.00 & 0.00 & 0.00 & 0.00 & 2.00 & 0.00 & 0.00 & 0.00 \\
\hline Zircon & $\mathrm{Zrc}$ & 0.00 & 0.00 & 0.00 & 0.00 & 0.00 & 0.00 & 1.00 & 0.00 & 0.00 & 0.00 & 1.00 & 0.00 & 0.00 & 0.00 \\
\hline Vlasovite & Vla & 2.00 & 0.00 & 0.00 & 0.00 & 0.00 & 0.00 & 1.00 & 0.00 & 0.00 & 0.00 & 4.00 & 0.00 & 0.00 & 0.00 \\
\hline \multicolumn{16}{|c|}{ Non-HFSE phases } \\
\hline Analcime & Anl & 1.00 & 0.00 & 0.00 & 0.00 & 0.00 & 0.00 & 0.00 & 0.00 & 0.00 & 1.00 & 2.00 & 2.00 & 0.00 & 0.00 \\
\hline Aegirine & Aeg & 1.00 & 0.00 & 0.00 & 0.00 & 1.00 & 0.00 & 0.00 & 0.00 & 0.00 & 0.00 & 2.00 & 0.00 & 0.00 & 0.00 \\
\hline Pectolite & Pct & 1.00 & 0.00 & 2.00 & 0.00 & 0.00 & 0.00 & 0.00 & 0.00 & 0.00 & 0.00 & 3.00 & 1.00 & 0.00 & 0.00 \\
\hline Annite & $\mathrm{Ann}_{\mathrm{bi}}$ & 0.00 & 1.00 & 0.00 & 3.00 & 0.00 & 0.00 & 0.00 & 0.00 & 0.00 & 1.00 & 3.00 & 2.00 & 0.00 & 0.00 \\
\hline Fluorite & $\mathrm{Fl}$ & 0.00 & 0.00 & 1.00 & 0.00 & 0.00 & 0.00 & 0.00 & 0.00 & 0.00 & 0.00 & 0.00 & 0.00 & 2.00 & 0.00 \\
\hline \multicolumn{16}{|c|}{ Melt/fluid components } \\
\hline $\mathrm{Na}_{2} \mathrm{SiO}_{3}$ & Nms & 2.00 & 0.00 & 0.00 & 0.00 & 0.00 & 0.00 & 0.00 & 0.00 & 0.00 & 0.00 & 1.00 & 0.00 & 0.00 & 0.00 \\
\hline & $\mathrm{CaO}$ & 0.00 & 0.00 & 1.00 & 0.00 & 0.00 & 0.00 & 0.00 & 0.00 & 0.00 & 0.00 & 0.00 & 0.00 & 0.00 & 0.00 \\
\hline & $\mathrm{H}_{2} \mathrm{O}$ & 0.00 & 0.00 & 0.00 & 0.00 & 0.00 & 0.00 & 0.00 & 0.00 & 0.00 & 0.00 & 0.00 & 2.00 & 0.00 & 0.00 \\
\hline
\end{tabular}

*Average of 118 EGM core analyses. 


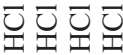

的的尔

०००0.

$+++$

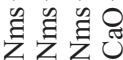

ส้ปัป ป

$0-10$

o $0^{m} 0^{m}$

서넌

这这究

$\infty \infty 00$

0ं $0 \dot{0}$

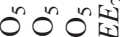

त. तु

乙二 Z品

む寸す。

$0 \dot{0} 0+$

$+++0^{n}$

나응

$\circ \circ 00$

है

完是是是

동ㄷํㅇ

웅웅ㅇ

$+++$

范芯吉

ต

$+\dot{+}+0$

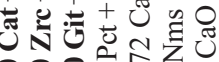

8용ำ

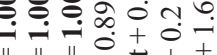

\| $\|+\vec{*}++$

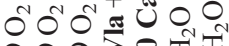

으으룓ㄴ

뭉요유. 눙

$+++1100$

○ช

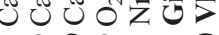

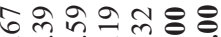

- -0 i

+ t t o o o l a

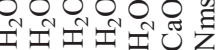

유엥ㅇㅇ

Ni०0.

+
+++
+

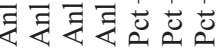

증ㅎํㄴํㅇㅇㅇ

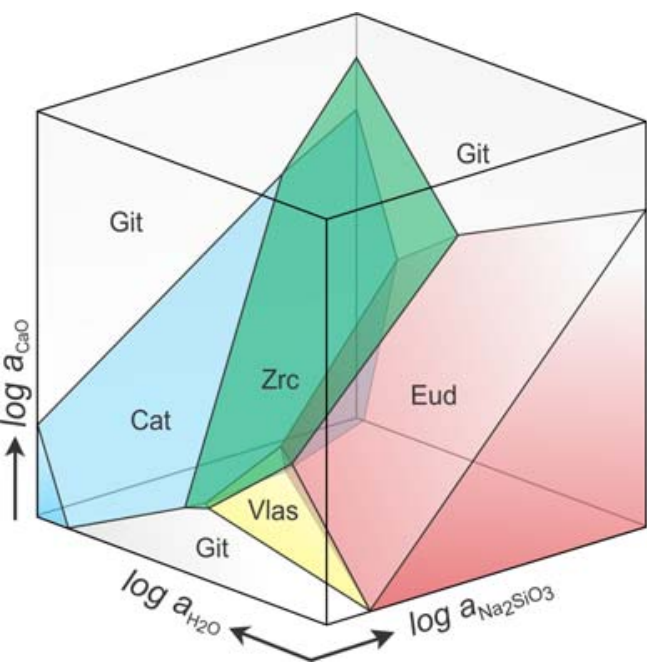

FIG. 7. Semi-quantitative 3D petrogenetic grid in $\log a_{\mathrm{Nms}}$ $-\log a_{\mathrm{H}_{2} \mathrm{O}}-\log a_{\mathrm{CaO}}$ space illustrating the relative stability volumes for the $\mathrm{Na}-\mathrm{Ca}$ zirconosilicates eudialyte (red), catapleiite (blue), gittinsite (grey transparent), zircon (green) and vlasovite (yellow). The divariant reactions, calculated for the system of $\mathrm{NaO}_{0.5}-\mathrm{KO}_{0.5}-\mathrm{CaO}-\mathrm{FeO}-$ $\mathrm{REEO}_{1.5}-\mathrm{ZrO}_{2}-\mathrm{TiO}_{2}-\mathrm{NbO}_{2.5}-\mathrm{AlO}_{1.5}-\mathrm{SiO}_{2}-\mathrm{HO}_{0.5}-\mathrm{FO}_{-0.5}$ $\mathrm{ClO}_{-0.5}$ (Table $5 a, b$ ), each define a 2-phase stability plane in 3D $\log$ activity space. The stability field for gittinsite overlies all other stability fields at high $\log a_{\mathrm{CaO}}$, low $\log$ $\mathrm{a}_{\mathrm{H}_{2} \mathrm{O}}$ and $\operatorname{low} \log a_{\mathrm{Nms}}$. Divariant planes between gittinsite and other phases are left semi-transparent to illustrate the 3D geometry of the stability volumes below.

the kakortokites. The stability of vlasovite in the kakortokites may be limited additionally by the relatively low $T$ during hydrothermal alteration $\left(<450^{\circ} \mathrm{C}\right)$, i.e. in Si-saturated systems vlasovite typically represents a high $T$ assemblage $\left(>600^{\circ} \mathrm{C}\right.$ at 1 kbar, e.g. Currie and Zaleski, 1985; Marr and Wood, 1992; Birkett et al., 1992).

\section{Alteration of EGM and catapleiite to gittinsite}

The gittinsite stability volume (grey transparent field, Fig. 7) overlies all other fields at high log $a_{\mathrm{CaO}}$. Based on the abundance of analcime, the presence of a crosscutting hydrothermal vein and the fact that gittinsite overprints a catapleiite assemblage, we assume that gittinsite formation also occurred at elevated levels of water activity, i.e. most probably in the presence of a free fluid phase. This can be illustrated with a vertical 2D section at high $\log a_{\mathrm{H}_{2} \mathrm{O}}$ (Fig. 9). Assuming constant water activity, gittinsite can stabilize after catapleiite following: (1) a reduction in alkalinity; (2) an increase in $\mathrm{CaO}$ activity; or (3) a combination of 

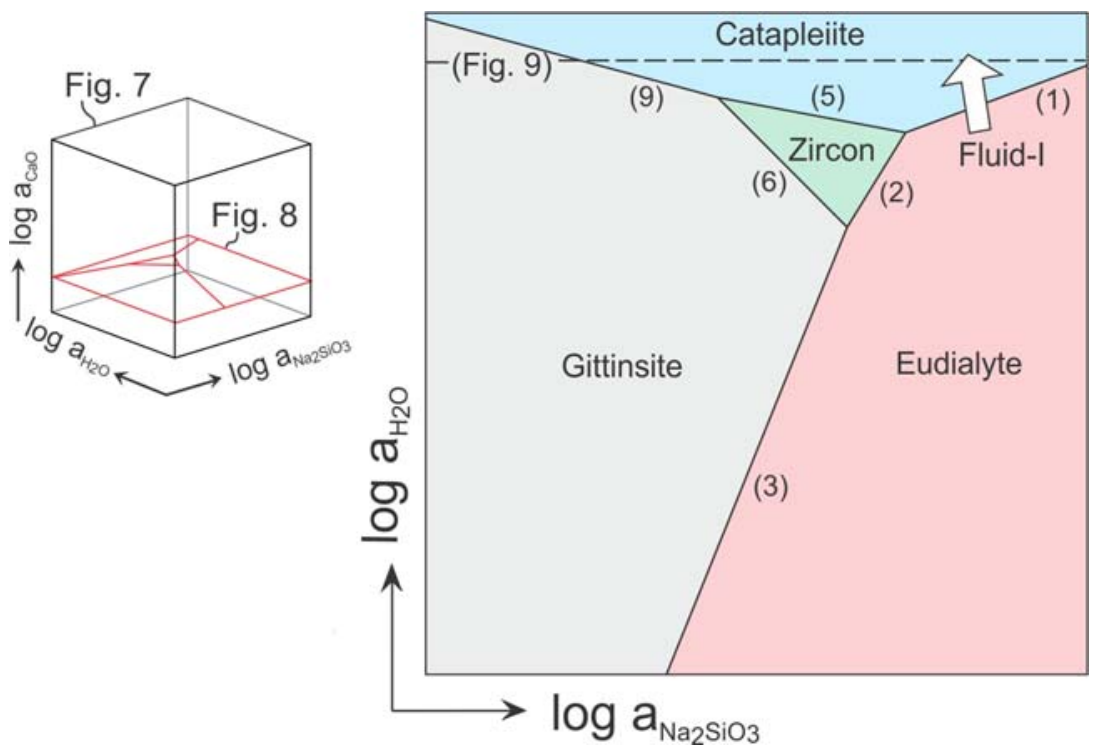

FIG. 8. Horizontal section through the petrogenetic grid in Fig. 7, illustrating the relative stability fields of EGM and alteration products as a function of $\log a_{\mathrm{H}_{2} \mathrm{O}}$ and $\log a_{\mathrm{Nms}}$ at constant $\mathrm{CaO}$ activity. Catapleiite formation is governed by increasing $\mathrm{a}_{\mathrm{H}_{2} \mathrm{O}}$ (arrow, Fluid I). The relative location with respect to Fig. 7 is indicated on the right and lies just above the vlasovite stability volume (see text for details). Lines are labelled with reaction numbers in Table $5 b$. The dashed line marks the location of the vertical section in Fig. 9, which represents a theoretical level of water saturation.

both (Fluid-II in Fig. 9). Because of abundant pectolite and fluorite in the gittinsite-paragenesis and the rare occurrence of this paragenesis in general, we infer that an overall input of calcium was required to form gittinsite at the expense of EGM and catapleiite. This was probably associated with a simultaneous decrease in alkalinity, thereby avoiding the zircon stability field prior to gittinsite stabilization (Fluid-II, Fig. 9). Direct replacement of EGM by gittinsite without the intermediate step of catapleiite formation (reaction 3 in Table $5 b$ and Fig. 8) requires an increase in $a_{\mathrm{CaO}}$ or decrease in $a_{\mathrm{Nms}}$ at locally reduced water activity, and probably reflects incomplete equilibration of EGM at higher water activities. Rims of $\mathrm{Sr}$-rich secondary EGM in the gittinsite paragenesis (Fig. $5 f, g, h$ ) suggest that this fluid also added $\mathrm{Sr}$.

Both $\mathrm{Ca}$ and $\mathrm{Sr}$ are depleted with continued magmatic evolution, i.e. of all intrusive units, the augite syenite contains the highest concentrations of $\mathrm{Ca}$ and $\mathrm{Sr}$ (c. 3-6 wt.\% and 300-400 ppm, respectively; Bailey et al., 2001). As such, alteration of earlier magmatic units, such as the augite syenites entrained as autoliths in unit +3 (Fig. 2) where gittinsite was found, could provide a potential source for $\mathrm{Ca}$ - and $\mathrm{Sr}$-bearing fluids. Alternative mechanisms for a relative increase in
$\mathrm{CaO}$ activity and/or decrease in alkalinity within internal fluids may involve late- to post-magmatic breakdown of other Ca-bearing phases, such as apatite or fluorite, or segregation of complementary alkali-rich and alkali-poor fluids/vapours (e.g. Shmulovich and Churakov, 1998; Mark1 and Baumgartner, 2002). Such processes, however, may not satisfactorily explain the addition of $\mathrm{Sr}$. The extremely rare occurrence of gittinsite in the kakortokites and agpaitic rocks in general is probably related to the overall high alkalinity, and the high buffering capacity of $\mathrm{Ca}$ in F-rich hydrothermal environments by precipitation of fluorite and phases like nacareniobsite-(Ce).

Limited evidence for $\mathrm{Ca}$ metasomatism by external fluids at Ilímaussaq includes a study of endo-skarn assemblages containing Ca-rich garnet, epidote (or allanite) and ilvaite (Graser and Markl, 2008). These assemblages were suggested to have formed from seawater which percolated through the overlying basalts and sandstones and entered the complex along fractures and faults upon cooling. Although the gittinsite-sample was found at a considerable distance from the margin of the complex, such a process could explain the input of both $\mathrm{Ca}$ and Sr. Calcic metasomatism has also been suggested as the main mechanism for 


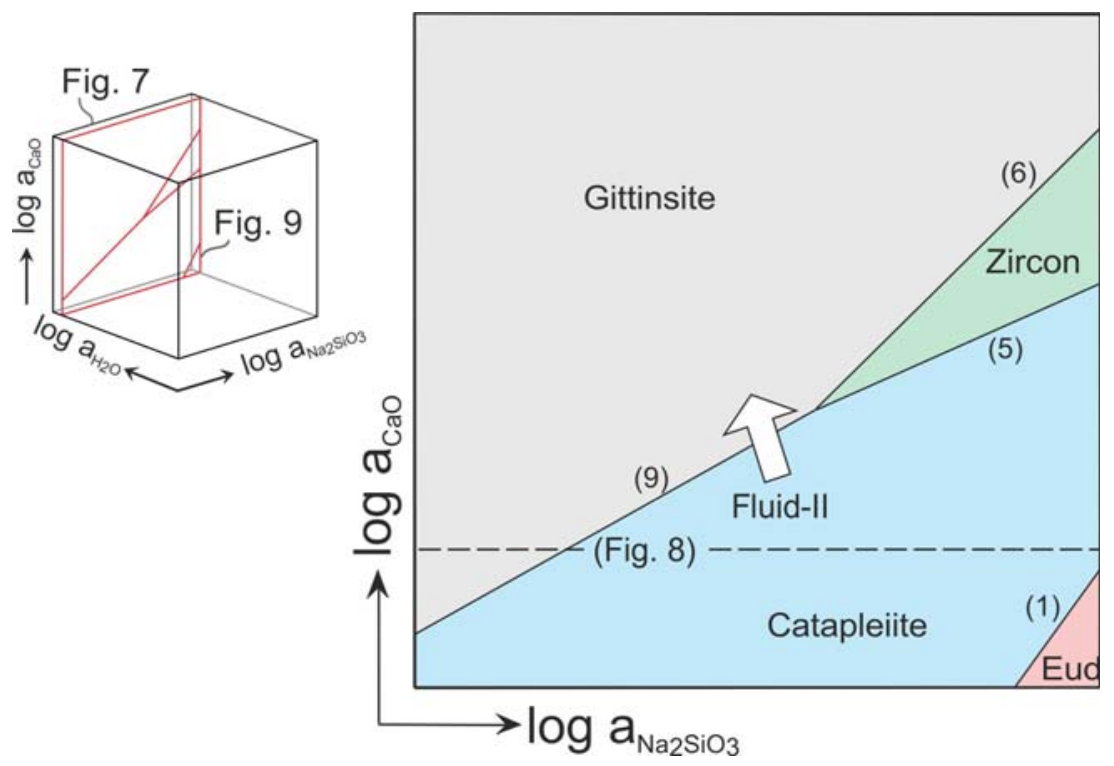

FIG. 9. Vertical section through the petrogenetic grid in Fig. 7, illustrating the relative stability fields of EGM and alteration products as a function of $\log a_{\mathrm{CaO}}$ and $a_{\mathrm{Nms}}$ at constant $a_{\mathrm{H}_{2} \mathrm{O}}$. Lines are labelled with reaction numbers in Table $5 b$. Gittinsite formation at the expense of catapleiite at high $\mathrm{H}_{2} \mathrm{O}$ activity (or saturation) can be achieved by decreasing $a_{\mathrm{Nms}}$ and increasing $a_{\mathrm{CaO}}$ (arrow, Fluid-II).

gittinsite formation in some peralkaline granites (sometimes rock forming, e.g. Strange Lake), where gittinsite commonly replaces zircon or elpidite $\left(\mathrm{Na}_{2} \mathrm{ZrSi}_{6} \mathrm{O}_{15} \cdot 3 \mathrm{H}_{2} \mathrm{O}\right)$ together with armstrongite $\left(\mathrm{CaZrSi}_{6} \mathrm{O}_{15} \cdot 3 \mathrm{H}_{2} \mathrm{O}\right)$ and quartz (i.e. highSi zirconosilicate assemblages; Marks et al., 2011). In these systems, Ca-metasomatism was associated with significant hydrothermal remobilization of primary $\mathrm{Zr}, \mathrm{Nb}$ and $R E E$ and involved mixing of external Ca-rich fluids with late-magmatic F-rich fluids (e.g. Salvi and Williams-Jones, 1996; 2005; 2006; Kynicky et al., 2011).

\section{Alteration of EGM to zircon}

The zircon stability volume (green, Fig. 7) is bounded by EGM at high $a_{N m s}$ and low $a_{C a O}$, catapleiite at high $a_{\mathrm{H}_{2} \mathrm{O}}$ and gittinsite at high $a_{\mathrm{CaO}}$ and lower $a_{\mathrm{H}_{2} \mathrm{O}}$. The diagram suggests that an increase in $a_{\mathrm{CaO}}$ during gittinsite formation could also lead to zircon formation after both EGM and catapleiite. The fact that zircon was not observed in EGM alteration assemblages of the main kakortokites may suggest that the zircon field is smaller than currently presented in Fig. 7, or simply absent due to variations in temperature, pressure, $\mathrm{Si}$ activity or other parameters not considered here. In that case, the zircon-stability volume is replaced by a univariant line between catapleiite, gittinsite and EGM. The general topology in Fig. 7, however, implies that the zircon-bearing assemblages do not reflect a continuous alteration sequence by progressive fluid-rock reactions, but rather reflect localized and separate melt/fluid conditions which were achieved only in pegmatitic and more evolved/altered agpaitic units.

\section{Mass-balance and volume relations}

The observed and reported EGM decomposition assemblages from Ilímaussaq suggest that most elements were retained within the precursor EGM domains, with $\mathrm{Zr}$ sequestered in catapleiite, gittinsite or zircon, $\mathrm{Nb}$ in nacareniobsite-(Ce), fergusonite- $(\mathrm{Y})$ and other $\mathrm{Nb}$ phases, and $\mathrm{Fe}$ in aegirine. Nacareniobsite-(Ce) additionally acts as a major host for released $R E E$, while the remaining $R E E$ form fine-grained $\mathrm{Ca}-(\mathrm{Ba})-R E E$ silicates $(\mathrm{A} 1)$, or alternatively monazite when additional apatite is present. Using the most common type-I alteration assemblage (i.e. Cat $+\mathrm{Aeg}+\mathrm{Anl}+\mathrm{Ncr}+\mathrm{Ca}$-rich $\mathrm{A} 1+\mathrm{Fl}$, adapting a simplified EGM formula and treating $R E E$ as a single component) the alteration reaction can be expressed by the mass- and volume- 
balanced equation (abbreviations as in Fig. 3):

Eudialyte $s . l .+$ Fluid A

$\rightarrow$ Pseudomorph assemblage + Fluid B

$\mathrm{Na}_{14.35} R E E_{0.5} \mathrm{Ca}_{6} \mathrm{Fe}_{3} \mathrm{Zr}_{3} \mathrm{Nb}_{0.25} \mathrm{Si}_{25.25} \mathrm{O}_{74}$. $44(\mathrm{OH}) \mathrm{nCl}(\mathrm{Eud})$

$+8.79 \mathrm{~F}_{\text {Liq }}^{-}+3 \mathrm{Al}_{\text {Liq }}^{3+}+7 \mathrm{H}_{2} \mathrm{O}_{\text {Liq }}$

$\rightarrow 3 \mathrm{Na}_{1.2} \mathrm{Ca}_{0.4} \mathrm{ZrSi}_{3} \mathrm{O}_{9} \mathrm{n} 2\left(\mathrm{H}_{2} \mathrm{O}\right)(\mathrm{Cat})$

$+3 \mathrm{NaFeSi}_{2} \mathrm{O}_{6}(\mathrm{Aeg})$

$+3 \mathrm{NaAlSi}_{2} \mathrm{O}_{6} \cdot \mathrm{H}_{2} \mathrm{O}(\mathrm{Anl})$

$+0.25 \mathrm{Na}_{3} \mathrm{Ca}_{3}(R E E) \mathrm{Nb}\left(\mathrm{Si}_{2} \mathrm{O}_{7}\right)_{2} \mathrm{OF}_{3}(\mathrm{Ncr})$

$+0.04 \mathrm{HCa}_{3} R E E_{6}\left(\mathrm{SiO}_{4}\right)_{6}(\mathrm{~F}),(\mathrm{Al})+4 \mathrm{CaF}_{2}(\mathrm{Fl})$

$+2 \mathrm{Na}_{2} \mathrm{O}_{\text {Liq }}+3 \mathrm{SiO}_{2 \mathrm{Liq}}+\mathrm{Cl}_{\text {Liq }}^{-}$

(reaction 1)

Note that the equation is not charge-balanced and semi-balanced for $\mathrm{O}$ and $\mathrm{H}$ due to the unconstrained fluid speciation and volatile contents of EGM. The growth of catapleiite, aegirine and nacareniobsite-(Ce) is confined by the availability of $\mathrm{Zr}, \mathrm{Fe}$ and $\mathrm{Nb}$, respectively. Their theoretical relative volumes within the pseudomorph volume can be calculated using the Z-values and unit-cell volumes, yielding proportions of $40 \%, 18 \%$ and $5 \%$ for catapleiite, aegirine and nacareniobsite(Ce), respectively. These are in good agreement with visual estimates in most pseudomorph assemblages (Fig. 3g,h). Residual REE are contained within A1 (unit-cell volumes unknown), while $\mathrm{Ca}$ re-precipitated as fluorite by addition of $\mathrm{F}$. If all $\mathrm{Ca}$ is retained in this way, fluorite should account for $\sim 9 \%$ of the pseudomorph volume. The proportions of fluorite, however, rarely exceed $5 \%$. Some of the remaining $\mathrm{Ca}$ may be used to form pectolite or apatite, or is lost from the EGM micro-domains. The absence of secondary $\mathrm{Cl}$ phases in or around the pseudomorphs, i.e. no sodalite rims on primary nepheline (as observed in the Igaliko and Sushina complexes; Finch, 1991 and Mitchell and Chakrabarty, 2012, respectively), implies that all $\mathrm{Cl}$ was released to the fluid phase.

In all pseudomorph assemblages the remaining interstitial volume is occupied by analcime, albite and/or K-feldspar, requiring significant input of aluminum. In reaction 1 , the amount of analcime is calculated based on a remaining pseudomorph volume of $27 \% \quad(\mathrm{Cat}+\mathrm{Aeg}+\mathrm{Ncr}+\mathrm{Fl}=73 \%$, neglecting A1). A similar calculation for albitedominated assemblages requires $2.5 \mathrm{~mol}$ of albite per mol of replaced EGM to fill $27 \%$ of the total pseudomorph volume. This reduces the required input of $\mathrm{Al}$ and $\mathrm{H}_{2} \mathrm{O}$ to 2.5 and 4 mol., respectively. Although $\mathrm{Al}$ is often considered to be immobile during fluid-rock interactions, it was undoubtedly soluble in the system considered here. A potential source of $\mathrm{Al}$ is the associated constant-volume replacement of primary nepheline and alkali feldspar by analcime (Markl and Baumgartner, 2002). As EGM contain very little $\mathrm{P}$ and $\mathrm{Ba}$, these elements must additionally be added to form EGM alteration assemblages containing monazite, apatite, britholite(Ce) and Ba-REE silicates. The restricted occurrence of these assemblages suggests that $\mathrm{P}$ and $\mathrm{Ba}$ contents in the fluids were highly variable, which may reflect local interaction of the fluids with (or precipitation of) phases enriched in these elements, e.g. apatite or K-feldspar.

In summary, the observed relative proportions combined with estimated volumes of secondary phases from reaction 1 suggest that the alteration of EGM to catapleiite-type assemblages was not associated with significant remobilization of REE, $\mathrm{Zr}$ and $\mathrm{Nb}$. The reaction reflects a net uptake of $\mathrm{F}$, $\mathrm{H}_{2} \mathrm{O}, \mathrm{Al}$, and occasionally $\mathrm{P}$ and $\mathrm{Ba}$ from the fluid phase, whereas $\mathrm{Na}, \mathrm{Si}$ and $\mathrm{Cl}$ were lost from the assemblage.

The sequential alteration of EGM, first to catapleiite and then to gittinsite, is associated with a significant volume reduction. With preservation of $\mathrm{Zr}$, gittinsite consumes $57 \%$ of the replaced catapleiite volume, and only $23 \%$ of the total EGM volume. This again correlates well with the observed proportions of gittinsite and the comparatively large proportion of analcime-filled 'pore space' in the gittinsite-bearing pseudomorphs (Fig. 5). In terms of REE, however, the gittinsitebearing pseudomorphs contain relatively small proportions of $R E E$-bearing phases in general, and a comparably high proportion of heavy rare-earth element (HREE)/Y-bearing phases (Fergusonite-(Y) and unidentified Ca-HREE-Y minerals, Fig. $5 c, d)$ with respect to the catapleiitetype paragenesis. This could suggest that the gittinsite-forming fluid was more capable of fractionating the light and heavy $R E E$, potentially reflecting a difference in $\mathrm{pH}$ and/or $\mathrm{F}$ and $\mathrm{Cl}$ contents between the two fluids.

\section{Mobility of HFSE and REE and comparison to peralkaline granites}

Although HFSE and REE are generally considered to be highly immobile in hydrothermal systems, 
studies of HFSE-REE mineralization in peralkaline granites and syenites such as Strange Lake, Thor Lake, Tamazeght, Khan Bogd and Khaldzan Buregte have shown that late-magmatic hydrothermal fluids enriched in ligands such as $\mathrm{Cl}^{-}, \mathrm{F}^{-}$, $\mathrm{PO}_{4}^{3-}, \mathrm{SO}_{4}^{2-}$ and $\mathrm{OH}^{-}$can lead to significant remobilization of primary HFSE and REE (e.g. Boily and Williams-Jones, 1994; Salvi and Willliams-Jones, 1996; 2005; 2006; Salvi et al., 2000; Kynicky et al., 2011; Sheard et al., 2012; Gysi and Williams-Jones, 2013). Recent numerical and experimental results have shown that $R E E$ (especially light rare-earth elements (LREE)) are most effectively transported as $\mathrm{Cl}$ complexes (e.g. Migdisov et al., 2009; Migdisov and Williams-Jones; 2014), whereas $\mathrm{Zr}$ and $\mathrm{Nb}$ remobilization is most effective via complexation with mixed F-OH compounds (e.g. Migdisov et al., 2011; Timofeev et al., 2015).

It is clear that most EGM alteration in the kakortokites occurred by aqueous fluids carrying significant amounts of $\mathrm{F}$ and $\mathrm{Cl}$, the latter being released to the fluid upon EGM breakdown. By analogy with the peralkaline granites listed above, this could provide a potential mechanism for significant hydrothermal remobilization of the primary REE-HFSE. However, in the presence of $\mathrm{F}$, the mobility of REE-chloride complexes is strongly limited to fluids with low $\mathrm{pH}\left(<4\right.$ at $\left.400^{\circ} \mathrm{C}\right)$ due to precipitation of insoluble $R E E$-fluoride phases at higher $\mathrm{pH}$ (e.g. Migdisov et al., 2009; Migdisov and Williams-Jones, 2014; Gysi and Williams-Jones, 2013). Markl and Baumgartner (2002) argued that late-stage fluids in Ilímaussaq are characterized by neutral to high $\mathrm{pH}$ and that the $\mathrm{pH}$ is strongly controlled by the $\mathrm{Na}$ to $\mathrm{Cl}$ ratio $(\mathrm{pH}>8$ at $\mathrm{Na} / \mathrm{Cl}>1$ ). With the inferred insolubility of $R E E-\mathrm{F}$ species at neutral to high $\mathrm{pH}$ (Migdisov and Williams-Jones, 2014), the released REE, Nb and $\mathrm{Ca}$ from the EGM micro-domains would immediately react with fluorine from the fluid to form fluorite, A1 and nacareniobsite-(Ce). This would subsequently lead to a decrease in the $\mathrm{F}^{-}$activity of the fluid, and thereby also decrease the potential mobility of $\mathrm{Zr}$ and $\mathrm{Nb}$ as fluoride and hydroxyl-fluoride complexes, even if such complexes would be stable at the considered temperature and $\mathrm{pH}$ (Migdisov et al., 2011; Timofeev et al., 2015).

The influence of $\mathrm{pH}$ on the solubility of REEchloride and -fluoride complexes (e.g. Migdisov et al., 2009; Migdisov and Williams-Jones, 2014; Gysi and Williams-Jones, 2013) may be crucial in understanding why fluid-driven remobilization of
HFSE and REE is relatively ineffective with respect to orthomagmatic enrichment processes in highly Si-undersaturated and persodic systems such as the kakortokites, as opposed to inherently more acidic systems such as the peralkaline granites of Thor Lake, Strange Lake and Khan Bogd (e.g. Sheard et al., 2012; Gysi and Williams-Jones, 2013; Kynicky et al., 2011) or related skarn-deposits (e.g. Ambohimirahavavy complex in Madagascar, Estrade et al., 2015). In granitic systems, magmatic fluids enriched in $\mathrm{F}$ and $\mathrm{Cl}$ are more likely to evolve towards low $\mathrm{pH}$ and may thus be more capable of remobilizing HFSE and REE and concentrating them to potentially economic levels when magmatic mineralization processes are insufficient.

\section{Conclusions and economic implications}

Pseudomorph assemblages after primary eudialytegroup minerals in the kakortokites of the Ilímaussaq complex are invariably dominated by the $\mathrm{Na}$ zirconosilicate catapleiite. The catapleiiteassemblages record an overall increase in $\mathrm{H}_{2} \mathrm{O}$ activities, which reflects the presence of $\mathrm{Na}-, \mathrm{Cl}-$ and F-rich aqueous fluids at the final stages of kakortokite crystallization following a nearcontinuous evolution from melt to fluid. EGM alteration is slightly more pervasive in the TLK sequence and lujavrites than in the main LLK sequence, which probably reflects higher volatile contents in the more evolved melts from which they crystallized. The pseudomorph assemblages generally require an input of $\mathrm{H}_{2} \mathrm{O}, \mathrm{Al}$ and $\mathrm{F}$, and occasionally $\mathrm{Ba}$ and $\mathrm{P}$, to the EGM micro-domains, whereas $\mathrm{Cl}$ was released to the fluid phase. Zirconbearing EGM alteration assemblages were not encountered in the current sample set, confirming their restricted occurrence to pegmatitic and more strongly altered units of the complex (Karup-Møller et al., 2010). Zircon formation is therefore interpreted to record localized melt-fluid evolution in units not representative for the main kakortokite body. Similarly, stabilization of the rare $\mathrm{Ca}$ zirconosilicate gittinsite, associated with analcime, pectolite, fluorite, annite, $\mathrm{Ca}-R E E$ silicates and allanite-(Ce), was triggered by a local increase in $\mathrm{CaO}$ activity along with a complementary decrease in alkalinity and is inferred to result from a separate, low $T$ post-magmatic alteration event by a Ca- and Sr-bearing aqueous fluid.

Alteration of EGM has primarily affected the HFSE and REE-mineralization through increased 
dissemination and fractionation of the prospective elements among the secondary minerals, while leaving the overall ore grade and resources unaffected. We infer that the immobile behaviour of HFSE and REE during autometasomatic alteration of EGM in the kakortokites relates to a relatively high $\mathrm{pH}(<8)$ of the late-magmatic fluids, triggering immediate precipitation of F-bearing phases such as fluorite, nacareniobsite-(Ce) and $\mathrm{Ca}$ $R E E$ silicates (A1) upon interaction of fluorine with the released $\mathrm{Ca}, R E E$ and $\mathrm{Nb}$. The resulting decrease in $\mathrm{F}^{-}$activity would subsequently prevent the potential mobility of $\mathrm{Zr}$ and $\mathrm{Nb}$ as $\mathrm{F}$ and mixed OH-F complexes. A somewhat lower $\mathrm{pH}$ for the post-magmatic gittinsite-forming fluid compared to the late-magmatic catapleiite-forming fluids could explain the minor fractionation between $L R E E$ and HREE by preferential remobilization of $L R E E$ as chloride-complexes.

Despite the fact that most HFSE and REE are retained within the pseudomorphs, the mineralogical changes associated with the wide-spread decomposition of EGM in the kakortokites led to an increased complexity of the mineralization. A significant reduction in grain size $(<10 \mu \mathrm{m})$ and different physical properties for the secondary $\mathrm{Zr}$, $\mathrm{Nb}$ and $R E E$ phases may complicate liberation or chemical separation of the metals from the rock. The latter was observed during dissolution studies of EGM concentrates from the Lovozero complex (Russia), where the presence of secondary $\mathrm{Na}-\mathrm{Zr}$ silicates resulted in low $\mathrm{Zr}$ recovery (Zakharov et al., 2011). As such, further studies on the mineralogical and physical properties of EGM alteration assemblages will be of great value for optimal beneficiation of the kakortokites and other EGM occurrences, and enhance their potential value as future ore deposits.

\section{Acknowledgements}

This research was carried out as part of AMB's $\mathrm{PhD}$ project with financial support from the Geocenter Denmark (project number 4/2014) and benefited from collaborations in the EURARE project, funded by the European Community's Seventh Framework Programme (FP7/2007-2013, grant 309373). The authors thank Henning Bohse for kindly hosting and assisting them during field sessions at Kringlerne, Muriel Erambert for help with EPMA analyses in Oslo and John Bailey for providing a collection of samples. Per Kalvig, Alexander Bartels, Lotte Larsen and Quinten van der Meer are thanked for fruitful discussions. Constructive comments from reviewers
Stefano Salvi, Ian Coulson and Principal Editor Roger Mitchell are gratefully acknowledged and contributed to a significantly improved manuscript.

\section{References}

Andersen, S., Bohsen, H. and Steenfelt, A. (1988) Geological map of Greenland 1: 20000 the southern part of the Ilímaussaq complex, South Greenland. Grønlands Geologiske Undersøgelse.

Andersen, T., Erambert, M., Larsen, A.O. and Selbekk, R. S. (2010) Petrology of nepheline syenite pegmatites in the Oslo Rift, Norway: Zirconium silicate mineral assemblages as indicators of alkalinity and volatile fugacity in mildly agpaitic magma. Journal of Petrology, 51(11), 2303-2325.

Andersen, T., Erambert, M., Larsen, A.O. and Selbekk, R. S. (2013) Petrology of nepheline syenites pegmatites in the Oslo Rift, Norway: $\mathrm{Zr}$ and $\mathrm{Ti}$ mineral assemblages in miaskitic and agpaitic pegmatites in the Larvik Plutonic Complex. Mineralogia, 44, 61-98.

Ansell, H.G., Roberts, A.C., Plant, A.G. and Stuman, B. D. (1980) Gittinsite, a new calcium zirconium silicate from the Kipawa Agpaitic Syenite Complex, Quebec. The Canadian Mineralogist, 18, 201-203.

Bailey, J.C., Gwodz, R., Rose-Hansen, J. and Sørensen, H. (2001) Geochemical overview of the Ilímaussaq alkaline complex, South Greenland. Geology of Greenland Survey Bulletin, 190, 35-53.

Birkett, T.C., Miller, R.R., Roberts, A.C. and Mariano, A. N. (1992) Zirconium-bearing minerals of the Strange Lake intrusive complex, Quebec-Labrador. The Canadian Mineralogist, 30, 191-205.

Bohse, H. and Andersen, S. (1981) Review of the stratigraphic divisions of the kakortokite and lujavrite in southern Ilímaussaq. Rapport Grønlands Geologisk Undersøgelse, 103, 53-62.

Bohse, H., Brooks, C.K. and Kunzendorf, H. (1971) Field observations on the kakortokites of the Ilímaussaq intrusion, South Greenland, including mapping and analyses by portable $\mathrm{X}$-ray fluorescence equipment for zirconium and niobium. Rapport Grønlands Geologiske Undersøgelse, 38, 43-00.

Boily, M. and Williams-Jones, A.E. (1994) The role of magmatic and hydrothermal processes in the chemical evolution of the Strange Lake plutonic complex, Quebec-Labrador. Contributions to Mineralogy and Petrology, 118, 33-47.

Cámara, F., Sokolova, E. and Hawthorne, F.C. (2011) From structural topology to chemical composition. XII. Titanium silicates: the crystal chemistry of rinkite $\mathrm{Na}_{2} \mathrm{Ca}_{4} \mathrm{REETi}\left(\mathrm{Si}_{2} \mathrm{O}_{7}\right)_{2} \mathrm{OF}_{3}$. Mineralogical Magazine, 75, 2755-2774.

Chakhmouradian, A.R. and Zaitsev, A.N. (2002) CalciteAmphibole-Clinopyroxene rock from the Afrikanda Complex, Kola Peninsula, Russia: mineralogy and a 
possible link to carbonatites. III. Silicate minerals. The Canadian Mineralogist, 40, 1347-1374.

Chakhmouradian, A.R. and Zaitsev, A.N. (2012) Rare Earth Mineralization in Igneous Rocks: Sources and Processes. Elements, 8(5), 347-354.

Chakrabarty, A., Pruseth, K.L. and Sen, A.K. (2012) Compositions and petrogenetic significance of eudialyte group minerals from Sushina, Purulia, West Bengal. Journal of the Geological Society of India, 79, 449-459.

Chakrabarty, A., Mitchell, R.H., Ren, M., Sen, A.K. and Pruseth, K.L. (2013) Rinkite, cerianite-(Ce), and hingganite-(Ce) in syenites gneisses from the Sushina Hill Complex, India: occurrence, compositional data and petrogenetic significance. Mineralogical Magazine, 77, 3137-3153.

Coulson, I.M. (1997) Post-magmatic alteration in eudialyte from the North Qôroq centre, South Greenland. Mineralogical Magazine, 61, 99-109.

Currie, K.L. and Zaleski, E. (1985) The relative stability of elpidite and vlasovite: a P-T indicator for peralkaline rocks. The Canadian Mineralogist, 23, 577-582.

Estrade, G., Salvi, S., Béziat, D. and Williams-Jones, A.E. (2015) The origin of skarn-hosted rare-metal mineralization in the Ambohimirahavavy alkaline complex, Madagascar. Economic Geology, 110(6), 1485-1513.

Ferguson, J. (1964) Geology of the Ilímaussaq alkaline intrusion, South Greenland. Part 1. Description of map and structure. Meddelelser om Grønland, 172, 1-81.

Finch, A.A. (1991) Conversion of nepheline to sodalite during subsolidus processes in alkaline rocks. Mineralogical Magazine, 55, 459-463.

Graser, G. and Markl, G. (2008) Ca-rich ilvaiteepidote-hydrogarnet endoskarns: a record of latemagmatic fluid influx into the persodic Ilímaussaq complex, South Greenland. Journal of Petrology, 49, 239-265.

Gysi, A.P. and Williams-Jones, A.E. (2013) Hydrothermal mobilization of pegmatite-hosted REE and $\mathrm{Zr}$ at Strange Lake, Canada: A reaction path model. Geochimica et Cosmochimica Acta, 122, 324-352.

Jarosewich, E. and Boatner, L.A. (1991) Rare earth element reference samples for electron microprobe analyses. Geostandards Newsletter, 15, 397-399.

Johnsen, O. and Grice, J.D. (1999) The crystal chemistry of the eudialyte group. The Canadian Mineralogist, 37, 865-891.

Johnsen, O., Ferraris, G., Gault, R.A., Grice, J.D., Kampf, A.R. and Pekov, I.V. (2003) The nomenclature of eudialyte-group minerals. The Canadian Mineralogist, 41, 785-794.

Karup-Møller, S., Rose-Hansen, J. and Sørensen, H. (2010) Eudialyte decomposition minerals with new hithero undescribed phases from the Ilímaussaq complex, South Greenland. Bulletin of the Geological Society of Denmark, 58, 75-88.
Karup-Møller, S. and Rose-Hansen, J. (2013) New data on eudialyte decomposition minerals from kakortokites and associated pegmatites of the Ilímaussaq complex, South Greenland. Bulletin of the Geological Society of Denmark, 61, 47-70.

Kempe, U., Götze, J., Dandar, S. and Haberman, D. (1999) Magmatic and metasomatic processes during formation of the Nb-Zr-REE deposits Khaldzan Buregte and Tsakhir (Mongolian Altai): Indications from a combined CL-SEM study. Mineralogical Magazine, 63, 165-177.

Khadem Allah, B., Fontan, F., Kader, M.M., Onchew, P. and Sørensen, H. (1998) Reactions between agpaitic nephelinitic syenitic melts and sedimentary carbonate rocks - exemplified by the Tamazeght complex, Morocco. Geochemistry International, 36, 569-581.

Konnerup-Madsen, J. (2001) A review of the composition and evolution of hydrocarbon gases during solidification of the Ilímaussaq alkaline complex, South Greenland. Geology of Greenland Survey Bulletin, 190, 159-166.

Krumrei, T.V., Villa, I.M., Marks, M.A.W. and Markl, G. (2006) $\mathrm{A}{ }^{40} \mathrm{Ar} /{ }^{39} \mathrm{Ar}$ and $\mathrm{U} / \mathrm{Pb}$ isotopic study of the Ilímaussaq complex, South Greenland: Implications for the ${ }^{40} \mathrm{~K}$ decay sonstant and for the duration of magmatic activity in a peralkaline complex. Chemical Geology, 227, 258-273.

Kynicky, J., Chakhmouradian, A.R., Xu, C., Krmicek, L. and Galiova, M. (2011) Distribution and evolution of zirconium mineralization in perkalkaline granites and associated pegmatites of the Khan Bogd complex, southern Mongolia. The Canadian Mineralogist, 49, 947-965.

Larsen, L.M. (1976) Clinopyroxenes and coexisting mafic minerals from the alkaline Ilímaussaq intrusion, South Greenland. Journal of Petrology, 17, 258-290.

Larsen, L.M. and Sørensen, H. (1987) The Ilímaussaq intrusion - progressive crystallization and formation of layering in an agpaitic magma. Pp. 473-488 in: Alkaline Igneous Rocks (J.G. Fitton and B.G.J. Upton, editors). Geological Society of London Special Publication, 30.

Le Maitre, R.W. (2003) Igneous Rocks: A Classification and Glossary of Terms, 2nd edition. Cambridge University Press, Cambridge, UK.

Lindhuber, M.J., Marks, M.A.W., Bons, P.D., Wenzel, T. and Markl, G. (2015) Crystal mat-formation as an igneous layering forming process: Textural and geochemical evidence from the 'lower layered' nepheline syenite sequence of the Ilímaussaq complex, South Greenland. Lithos, 224-225 and 295-309.

Lorenzen, J. (1884) Untersuchungen einiger Mineralien aus Kangerdluarsuk in Grönland. Zeitschrift für Kristallographie, 9, 243-254.

Markl, G. and Baumgartner, L. (2002) pH changes in peralkaline late-magmatic fluids. Contributions to Mineralogy and Petrology, 144, 331-346. 
Markl, G., Marks, M.A.W., Schwinn, G. and Sommer, H. (2001) Phase equilibrium constraints on intensive crystallization parameters of the Ilímaussaq Complex, South Greenland. Journal of Petrology, 42, 2231-2258.

Markl, G., Marks, M.A.W. and Frost, B.R. (2010) On the controls of oxygen fugacity in the generation and crystallization of peralkaline melts. Journal of Petrology, 51, 1831-1847.

Marks, M.A.W. and Markl, G. (2015) The Ilímaussaq Alkaline Complex, South Greenland. Pp. 649-691 in: Layered Intrusions (B. Charlier, O. Namur, R. Latypov and C. Tegner, editors). Springer Geology, Dordrecht, The Netherlands.

Marks, M.W., Hettman, K. and Schilling, J.T. (2011) The mineralogical diversity of alkaline igneous rocks: critical factors for the transition from miaskitic to agpaitic phase assemblages. Journal of Petrology, 52, 439-455.

Marr, R.A. and Wood, S.A. (1992) Preliminary petrogenetic grids for sodium and calcium zirconosilicate minerals in felsic peralkaline rocks: The $\mathrm{SiO}_{2}-\mathrm{Na}_{2} \mathrm{ZrO}$ and $\mathrm{SiO}_{2}-\mathrm{CaZrO}$, pseudobinary systems. American Mineralogist, 77, 810-820.

Migdisov, A.A. and Williams-Jones, A.E. (2014) Hydrothermal transport and deposition of the rare earth elements by fluorine-bearing aqueous liquids. Mineralium Deposita, 49, 987-997.

Migdisov, A.A., Williams-Jones, A.E. and Wagner, T. (2009) An experimental study of the solubility and speciation of the rare earth elements (III) in fluorideand chloride-bearing aqueous solutions at temperatures up to $300^{\circ} \mathrm{C}$. Geochimica et Cosmochimica Acta, 73, 7087-7109.

Migdisov, A.A., Williams-Jones, A.E., van Hinsberg, V. and Salvi, S. (2011) An experimental study of the solubility of baddelyite $\left(\mathrm{ZrO}_{2}\right)$ in fluoride-bearing solutions at elevated temperature. Geochimica et Cosmochimica Acta, 75, 7426-7434.

Mitchell, R.H. and Chakrabarty, A. (2012) Paragenesis and decomposition assemblage of a Mn-rich eudialyte from the Sushina peralkaline nepheline syenite gneiss, Paschim Banga, India. Lithos, 152, 218-226.

Mitchell, R.H. and Liferovich, R.P. (2006) Subsolidus deuteric/hydrothermal alteration of eudialyte in lujavrite from the Pilansberg alkaline complex, South Africa. Lithos, 91, 352-372.

Morimoto, N. (1988) Nomenclature of pyroxenes. Mineralogy and Petrology, 39, 55-76.

Olivo, G.R. and Williams-Jones, A.E. (1999) Hydrothermal REE-rich eudialyte from the Pilanesberg complex, South Africa. The Canadian Mineralogist, 37, 653-663.

Petersen, O.V., Rønsbo, J.G. and Leonardsen, E.S. (1989) Nacareniobsite-(Ce), a new mineral species from the Ilímaussaq alkaline complex, South Greenland, and its relation to mosandrite and the rinkite series. Neues Jahrbuch für Mineralogie Monatshefte, 2, 84-96.

Pfaff, K., Krumrei, T.V., Marks, M.A.W., Wenzel, T., Rudolf, T. and Markl, G. (2008) Chemical and physical evolution of the 'lower layered series' from the nepheline syenitic Ilímaussaq intrusion, South Greenland: Implications for the origin of magmatic layering in peralkaline felsic liquids. Lithos, 106, 280-296.

Pouchou, J.L. and Pichoir, F. (1984) A new model for quantitative X-ray microanalysis. I. Application to the analysis of homogeneous samples. Recherche Aerospatiale, 3, 13-38.

Ratschbacher, B.C., Marks, M.A.W., Bons, P.D., Wenzel, T. and Markl, G. (2015) Emplacement and geochemical evolution of highly evolved syenites investigated by a combined structural and geochemical field study: the lujavrites of the Ilímaussaq complex, SW Greenland. Lithos, 231, 62-76.

Rønsbo, J.G., Sørensen, H., Roda-Robles, E., Fontan, F. and Monchoux, P. (2013) Rinkite-nacareniobsite-(Ce) solid solution series and hainite from the Ilímaussaq alkaline complex: occurrence and compositional variation. Bulletin of the Geological Society Denmark, 62, 1-15.

Roskill (2011) Rare Earths and Yttrium: Market Outlook to 2015. 14th Edition. Roskill Information Services Ltd., London.

Salvi, S. and Williams-Jones, A.E. (1995) Zirconosilicate phase relations in the Strange Lake (Lac Brisson) pluton, Quebec-Labrador, Canada. American Mineralogist, 80, 1031-1040.

Salvi, S. and Williams-Jones, A.E. (1996) The role of hydrothermal processes in concentrating high-field strength elements in the Strange Lake peralkaline complex, northeastern Canada. Geochimica et Cosmochimica Acta, 60, 1917-1932.

Salvi, S. and Williams-Jones, A.E. (2005) Alkaline Granite-Syenite Deposits. Pp. 315-341 in: RareElement Geochemistry and Mineral Deposits (R.L. Linnen and I.M. Samson, editors). GAC Short Course Notes 17. Geological Association of Canada, St. John's, Canada.

Salvi, S. and Williams-Jones, A.E. (2006) Alteration, HFSE mineralisation and hydrocarbon formation in peralkaline igneous systems: Insights from the Strange Lake Pluton, Canada. Lithos, 91, 19-34.

Salvi, S., Fontan, F., Monchoux, P., Williams-Jones, A.E. and Moine, B. (2000) Hydrothermal mobilization of high field strength elements in Alkaline Igneous Systems: Evidence from the Tamazeght complex (Morocco). Economic Geology, 95, 559-576.

Schilling, J., Wu, F.Y., McCammon, C., Wenzel, T., Marks, M.A.W., Pfaff, K., Jacob, D.E. and Markl, G. (2011) The compositional variability of eudialyte-group minerals. Mineralogical Magazine, 75, 87-115. 
Sheard, E.R., Williams-Jones, A.E., Heiligmann, M., Pederson, C. and Trueman, D.L. (2012) Controls on the concentration of zirconium, niobium and the rare earth elements in the Thor Lake rare metal deposit, Northwest Territories, Canada. Economic Geology, 107, 81-104.

Shmulovich, K.I. and Churakov, S.V. (1998) Natural fluid phases at high temperature and low pressures. Journal of Geochemical Exploration, 62, 183-191.

Sokolova, E. and Hawthorne, F.C. (2008) From structural topology to chemical composition. V. Titanium silicates: crystal chemistry of nacareniobsite-Ce. The Canadian Mineralogist, 46, 1323-1342.

Stromeyer, F. (1819) Summary of meeting 16 December 1819. Göttingische gelehrte Anzeigen, 3, 1993-2000.

Sørensen, H. (1992) Agpaitic nepheline syenites: a potential source of rare elements. Applied Geochemistry, 7, 417-427.

Sørensen, H. (1997) The agpaitic rocks - an overview. Mineralogical Magazine, 61, 485-498.

Sørensen, H. (2001) Brief introduction to the Geology of the Ilímaussaq alkaline complex, South Greenland, and its exploration history. Geology of Greenland Survey Bulletin, 190, 7-24.
Sørensen, H., Bohse, H. and Bailey, J.C. (2006) The origin and mode of emplacement of lujavrites in the Ilímaussaq alkaline complex, South Greenland. Lithos, 91, 286-300.

Timofeev, A., Migdisov, A.A. and Williams-Jones, A.E. (2015) An experimental study of the solubility and speciation of niobium in fluoride-bearing aqueous solutions at elevated temperature. Geochimica et Cosmochimica Acta, 158, 103-111.

Upton, B.G.J. (2013) Tectono-magmatic evolution of the younger Gardar southern rift, South Greenland. Geological Survey of Denmark and Greenland Bulletin, 29, 1-128.

Ussing, N.V. (1912) Geology of the country around Julianehaab, Greenland. Meddelelser om Grønland, 38, 426.

Waight, T., Baker, J. and Willigers, B. (2002) Rb isotope dilution analyses by MC-ICPMS using $\mathrm{Zr}$ to correct for mass fractionation: towards improved $\mathrm{Rb}-\mathrm{Sr}$ geochronology? Chemical Geology, 186, 99-116.

Zakharov, V.I., Maiorov, D.V., Alishkin, A.R. and Matveev, V.A. (2011) Causes of insufficient recovery of zirconium during acidic processing of lovozero eudialyte concentrate. Russian Journal of Non-Ferrous Metals, 52, 423-428. 